\title{
HOM-HYPOREDUCTIVE TRIPLE ALGEBRAS
}

\section{SYLVAIN ATTAN ${ }^{1}$ and DONATIEN GAPARAYI ${ }^{2}$}

1Département de Mathématiques

Université d'Abomey-Calavi

01 BP 4521, Cotonou 01

Bénin

e-mail: syltane2010@yahoo.fr

${ }^{2}$ Ecole Normale Supérieure (E. N. S.)

BP 6983 Bujumbura

Burundi

e-mail: gapadona@yahoo.fr

\begin{abstract}
Hom-hyporeductive triple algebras are defined as a twisted generalization of hyporeductive triple algebras. Hom-hyporeductive triple algebras generalize right Hom-Lie-Yamaguti and right Hom-Bol algebras as the same way as hyporeductive triple algebras generalize right Lie-Yamaguti and right Bol algebras. It is shown that the category of Hom-hyporeductive triple algebras is closed under the process of
\end{abstract}

2020 Mathematics Subject Classification: 17A30, 17D99.

Keywords and phrases: hyporeductive triple algebra, Hom-Lie triple system, Hom-Bol algebra, Hom-Lie-Yamaguti algebra, Hom-hyporeductive triple algebra.

Received March 30, 2021

(C) 2021 Scientific Advances Publishers

This work is licensed under the Creative Commons Attribution International License (CC BY 3.0).

http://creativecommons.org/licenses/by/3.0/deed.en_US

Open Access (cc) (1)


taking nth derived binary-ternary Hom-algebras and by self-morphisms of binary-ternary algebras. Some examples of Hom-hyporeductive triple algebras are given.

\section{Introduction}

A hyporeductive triple algebra is a quadruple $(V, \cdot, *,\langle;\rangle$, consisting of a vector space $V$, bilinear maps $, *: V \times V \rightarrow V$ and a trilinear map $\langle;\rangle:, V \times V \times V \rightarrow V$ such that

(1) $x \cdot y=-y \cdot x$,

(2) $x * y=-y * x$,

(3) $\langle z ; y, x\rangle=-\langle z ; x, y\rangle$,

(4) $\sigma\{x \cdot(y \cdot z)-\langle x ; y, z\rangle\}=0$,

(5) $\sigma\{z *(x \cdot y)\}=0$,

(6) $\sigma\langle t ; z, x \cdot y\rangle=0$,

(7) $x \cdot\langle y ; z, t\rangle-y \cdot\langle x ; z, t\rangle+\langle y \cdot x ; z, t\rangle-\langle z * t ; y, x\rangle+\langle y * x ; z, t\rangle$

$-y *\langle x ; z, t\rangle+x *\langle y ; z, t\rangle-(z * t) *(y * x)-(z * t) \cdot(y * x)=0$,

(8) $u \cdot(x \cdot\langle y ; z, t\rangle-y \cdot\langle x ; z, t\rangle+\langle y \cdot x ; z, t\rangle)+\langle\langle u ; z, t\rangle ; y, x\rangle-\langle\langle u ; y$, $x\rangle ; z, t\rangle+\langle u ; y,\langle x ; z, t\rangle\rangle-\langle u ; x,\langle y ; z, t\rangle\rangle=0$,

(9) $u *(x \cdot\langle y ; z, t\rangle-y \cdot\langle x ; z, t\rangle+\langle y \cdot x ; z, t\rangle)=0$,

(10) $\langle v ; u, x \cdot\langle y ; z, t\rangle-y \cdot\langle x ; z, t\rangle+\langle y \cdot x ; z, t\rangle\rangle=0$,

(11) $x \cdot\langle y ; z, t\rangle-y \cdot\langle x ; z, t\rangle+\langle y \cdot x ; z, t\rangle+t \cdot\langle z ; y, x\rangle+\langle z \cdot t ; y, x\rangle=0$,

(12) $y *\langle x ; z, t\rangle-x *\langle y ; z, t\rangle+z *\langle t ; y, x\rangle-t *\langle z ; y, x\rangle=0$,

(13) $\sum\{\langle(\langle z \cdot t ; y, x\rangle+t \cdot\langle z ; y, x\rangle-z \cdot\langle t ; y, x\rangle) ; u, v\rangle+\langle u \cdot v ;\langle t ; y, x\rangle, z\rangle$ $+v \cdot\langle u ;\langle t ; y, x\rangle, z\rangle-u \cdot\langle v ;\langle t ; y, x\rangle, z\rangle-(\langle u \cdot v ;\langle z ; y, x\rangle, t\rangle+v \cdot\langle u ;\langle z ;$ $y, x\rangle, t\rangle-u \cdot\langle v ;\langle z ; y, x\rangle, t\rangle)\}=0$, 
(14) $\sum\{(\langle v ;\langle t ; y, x\rangle, z\rangle-\langle v ;\langle z ; y, x\rangle, t\rangle) * u+(\langle u ;\langle z ; y, x\rangle, t\rangle-\langle u ;\langle t$;

$y, x\rangle, z\rangle) * v\}=0$,

(15) $\sum\{\langle w ;(\langle v ;\langle t ; y, x\rangle, z\rangle-\langle v ;\langle z ; y, x\rangle, t\rangle), u\rangle+\langle w ;(\langle u ;\langle z ; y, x\rangle, t\rangle$

$-\langle u ;\langle t ; y, x\rangle, z\rangle), v\rangle\}=0$

where $\sigma$ denotes the sum over cyclic permutation of $x, y, z$ and $\sum$ is the one over $(z, t),(y, x)$, and $(u, v)$ for all $x, y, z, t, v, w \in V$. Hyporeductive algebras were introduced by Sabinin [22, 23], as an infinitesimal tool for the study of smooth hyporeductive loops which are a generalization of both smooth Bol loops and smooth reductive loops [22]. It is shown that the fundamental vector fields of any smooth hyporeductive loop constitute an algebra called hyporeductive algebra of vector fields. Further this notion has been extended to the one of abstract hyporeductive triple algebra [12, 13, 23] meaning a finite-dimensional vector space with two binary and one ternary operations satisfying some specific conditions. It turns out that hyporeductive triple algebras generalize right Bol algebras [20] and Lie triple algebras, i.e., LieYamaguti algebras [26]. The reader is referred to [11] for the classification of real two-dimensional hyporeductive triple algebras. The aim of this paper is a study of a Hom-type generalization of hyporeductive triple algebras. Roughly, a Hom-type generalization of kind of algebra is obtained by a certain twisting of the defining identities by a linear selfmap, called the twisting map, in such a way that when the twisting map is the identity map, then one recovers the original kind of algebra. In this scheme, e.g., associative algebras and Leibniz algebras are twisted into Hom-associative algebras and Hom-Leibniz algebras respectively [19] and, likewise, Hom-type analogues of alternative algebras, Jordan algebras or Malcev algebras are defined and discussed in [18, 27]. The Hom-type generalization of some classes of ternary algebras are discussed in $[3,30]$. One could say that the theory Hom-algebras originated in $[7,15,16]$ in a study of deformations of the Witt and Virasoro algebras (in 
fact, some $q$-deformations of the Witt and the Virasoro algebras have a structure of a Hom-Lie algebra [7]). The Hom-type generalization of binary algebras or ternary algebras is extended to the one of binaryternary algebras in $[4,5,10]$. Our present study of Hom-type generalization of Hyporeductive algebras is included in this setting.

A description of the rest of this paper is as follows:

In Section 2, we first recall some basics on Hom-algebras and then extend to binary-ternary Hom-algebras the notion of an $n$-th-derived (binary) Hom-algebra introduced in [27] and which generalizes the one introduced in [4]. Theorem 2.9 says that the category of right Hom-LieYamaguti algebras is closed under taking derived binary-ternary Homalgebras. Proposition 2.10 as well as Theorem 2.11 give a method for constructing a right Hom-Lie-Yamaguti algebra from a left Leibniz algebra and a Hom-Lie algebra respectively. Next, Theorem 2.15 allows the construction of a right Hom-Bol algebra from a left alternative algebra. In Section 3, we defined Hom-hyporeductive triple algebras and we point out that hyporeductive triple algebras are particular instances of Hom-hyporeductive triple algebras. Also Hom-hyporeductive triple algebras generalize right Hom-Bol and right Hom-Lie-Yamaguti algebras in the same way as hyporeductive triple algebras generalize right $\mathrm{Bol}$ and right Lie-Yamaguti algebras. Next we prove some construction theorems (Theorems 3.3, Corollary 3.4 and Corollary 3.5). The category of Homhyporeductive triple algebras is closed under self-morphisms (Theorem 3.3) and, subsequently, every hyporeductive triple algebra is twisted, along any self-morphism, into a Hom-hyporeductive algebra (Corollary 3.5). Theorem 3.6 says that the category of Hom-hyporeductive triple algebras is closed under taking derived binary-ternary Hom-algebras. In Section 4, relying on a classification of real 2-dimensional hyporeductive triple algebras given in [11], we classify all the algebra morphisms on all the real 2-dimensional hyporeductive triple algebras and then construct (for the case of nontrivial hyporeductive triple algebras) their associated Hom-hyporeductive triple algebras (applying thusly Corollary 3.5). 
Throughout this paper we will work over a ground field of characteristic 0 .

\section{Preliminaries and Some Results}

The main purpose of this section is to prove that any Leibniz (resp., alternative) algebra has a right Hom-Lie-Yamaguti (resp., right Hom-Bol) algebra structure and that right Hom-Lie-Yamaguti algebras are close under taking by $n$-th-derived binary-ternary Hom-algebras. We prove also that any Hom-Lie algebra has a right Hom-Lie-Yamaguti algebra structure.

Definition 2.1. Let $n \geq 2$, be an integer.

(i) An $n$-ary Hom-algebra $\left(A,[, \ldots],, \alpha=\left(a_{1}, \ldots, \alpha_{n-1}\right)\right)$ consists of a

vector space $A$, an $n$-linear map $[, \cdots]:, A^{\otimes n} \rightarrow A$ (the $n$-ary operation) and linear maps $\alpha_{i}: A \rightarrow A$ (the twisting maps), $i=1, \cdots, n-1$.

(ii) An $n$-ary Hom-algebra $(A,[, \cdots],, \alpha)$ is said to be multiplicative when the twisting maps $\alpha_{i}$ are all equals, $\alpha_{1}=\cdots=\alpha_{n-1}:=\alpha$ and $\alpha \circ[, \cdots]=,[, \cdots,] \circ \alpha^{\otimes n}$.

(iii) A linear map $f: A \rightarrow B$ of $n$-ary Hom-algebras is called a weak morphism if $f \circ[, \cdots,]_{A}=[, \cdots,]_{B} \circ f^{\otimes n}$. The weak morphism $f$ is called a morphism of the $n$-ary Hom-algebras $A$ and $B$ if $f \circ\left(\alpha_{i}\right)_{A}=\left(\alpha_{i}\right)_{B} \circ$ for $i=1, \cdots, n-1$.

Remark 2.2. If all $n-1$ twisting maps are the identity map $I d$ in an $n$-ary Hom-algebra $(A,[.],. \alpha)$, then it reduces to an usual $n$-ary algebra $(A,[, \cdots]$,$) . In this case, the weak morphism coincides with the$ morphism. 
If $n=2$ (resp., $n=3$ ), an $n$-ary Hom-algebra is called a binary, (resp., ternary) Hom-algebra. In the sequel, for our purpose and convenience, we shall consider only multiplicative Hom-algebras. HomLie algebras [7] constitute the first introduced class of (binary) Homalgebras.

Definition 2.3. A multiplicative Hom-algebra $(L, \cdot, \alpha)$ is called a Hom-Lie algebra if $x \cdot y=-y \cdot x$ (skew-symmetry) and the Hom-Jacobi identity $\sigma\{(x \cdot y) \cdot \alpha(z)\}=0$ holds for all $x, y, z \in L$. If $\alpha=I d$, a HomLie algebra reduces to Lie algebra.

Example 2.4 ([28]). There is a family of three-dimensional complex Hom-Lie algebras $\operatorname{sl}(2, \mathbb{C})_{\lambda}=\left(\operatorname{sl}(2, \mathbb{C}),[,]_{\alpha_{\lambda}}, \alpha_{\lambda}\right)(\lambda \neq 0)$ such that $\alpha_{\lambda}(e)=\lambda e, \alpha_{\lambda}(f)=\frac{1}{\lambda} f, \alpha_{\lambda}(h)=h$ and $[e, f]_{\lambda}=h,[h, f]_{\lambda}=-\frac{2}{\lambda} f,[h, e]_{\lambda}$ $=2 \lambda e$ with respect to a basis $(e, f, h)$, where $e=\left(\begin{array}{ll}0 & 1 \\ 0 & 0\end{array}\right), f=\left(\begin{array}{ll}0 & 0 \\ 1 & 0\end{array}\right)$, and $h=\left(\begin{array}{rr}1 & 0 \\ 0 & -1\end{array}\right)$. One can think of the collection $\left\{\left(\operatorname{sl}(2, \mathbb{C})_{\lambda}: \lambda \in \mathbb{C} \backslash\{0\}\right\}\right.$ as a one-parameter family of deformations of $\operatorname{sl}(2, \mathbb{C})$ into Hom-Lie algebras.

The class of ternary Hom-algebras that are of interest in our setting is the one of Hom-Lie-triple systems defined in [30]. Here, we consider a right Hom-Lie triple system instead of the left one which is called a HomLie triple system [30] for short. 
Definition 2.5. A right Hom-Lie-triple system is a multiplicative ternary Hom-algebra $(A,\langle\rangle,, \alpha)$ that satisfies:

$$
\begin{aligned}
\langle z ; y, x\rangle= & -\langle z ; x, y\rangle \text { (right skew-symmetry) } \\
\sigma\langle z ; y, x\rangle= & 0 \quad \text { (ternary Jacobi identity) } \\
\langle\langle w ; v, u\rangle ; \alpha(y), \alpha(x)\rangle= & \langle\langle w ; x, y\rangle ; \alpha(v), \alpha(u)\rangle+\langle\alpha(w) ;\langle v ; x, y\rangle, \alpha(u)\rangle \\
& +\langle\alpha(w) ; \alpha(v),\langle u ; x, y\rangle\rangle
\end{aligned}
$$

for all $x, y, u, v, w \in A$.

When $\alpha=I d$, we recover the usual notion of Lie triple systems [17]. The identity (1) is known as the ternary Hom-Nambu-Lie identity. Ternary Hom-algebras such as ternary Hom-Nambu algebras, ternary Hom-Nambu-Lie algebras and ternary Hom-Lie algebras are defined and studied in [3] and Hom-Jordan triple systems are studied in [30].

For binary Hom-algebras, the notion of an $n$-th-derived Hom-algebra is introduced and studied in [27] and extended and studied in binaryternary Hom-algebras case in [4]. Here, we give the definition of nthderived binary-ternary Hom-algebra for a binary-ternary Hom-algebra with two binary operations and one ternary operation.

Definition 2.6. Let $A:=\left(A,[,]_{1},[,]_{2},[],, \alpha\right)$ be a binary-ternary Hom-algebra and $n \geq 0$, an integer. Define on $A$ the $n$-th-derived binary operations $[,]_{1}^{(n)},[,]_{2}^{(n)}$ and the $n$-th-derived ternary operation $[,,]^{(n)}$ by $[,]_{1}^{(n)}:=\alpha^{2^{n-1}} \circ[,]_{1},[,]_{2}^{(n)}:=\alpha^{2^{n-1}} \circ[,]_{2}$ and $[,,]^{(n)}:=\alpha^{2^{n-1}} \circ[,$,$] . Then$ $A^{(n)}:=\left(A,[,]_{1}^{(n)},[,]_{2}^{(n)},[,,]^{(n)}, \alpha^{2^{n}}\right)$ will be called the $n$-th-derived (binary-ternary) Hom-algebra of $A$. 
We note $A^{(0)}=A, A^{(1)}=\left(A,[,]_{1}^{(1)}=\alpha \circ[,]_{1},[,]_{2}^{(1)}=\alpha \circ[,]_{2},[,]_{1}^{(1)}\right.$ $\left.=\alpha^{2} \circ[,],, \alpha^{2}\right)$.

One observes that if $[,]_{1}=0$ (or $[,]_{2}=0$ ), we get the notion of $n$-th-derived binary-ternary Hom-algebra defined in [4]. Furthermore, if $[,]=$,0 , we get the notion of $n$-th-derived binary Hom-algebra defined in [30].

Moving forward in general theory of Hom-algebras, a study of "binary-ternary" Hom-algebras is initiated in [10] by defining the class of Hom-Akivis algebras as a Hom-analogue of the class of Akivis algebras $[1,2,9]$. Then, another classes of binary-ternary Hom-algebras such as Hom-Lie-Yamaguti algebras [5] and Hom-Bol algebras [4] are introduced and studied and both generalize Hom-Lie triple systems.

In this paper, we consider only a right Hom-Lie-Yamaguti algebra instead the left one which is introduced and studied in [5] under the name Hom-Lie-Yamaguti algebra.

Definition 2.7. A right Hom-Lie-Yamaguti algebra is a quadruple $(L, \cdot,\langle,\rangle,, \alpha)$ in which $L$ is $\mathbb{K}$-vector space, "." is a binary operation and " $\langle,$,$\rangle " is a ternary operation on L$, and $\alpha: L \rightarrow L$ is a linear map such that

$$
\begin{aligned}
& \left(\mathrm{HLY}_{1}\right) \alpha(x \cdot y)=\alpha(x) \cdot \alpha(y), \\
& \left(\mathrm{HLY}_{2}\right) \alpha(\langle x ; y, z\rangle)=\langle\alpha(x) ; \alpha(y), \alpha(z)\rangle, \\
& \left(\mathrm{HLY}_{3}\right) x \cdot y=-y \cdot x, \\
& \left(\mathrm{HLY}_{4}\right)\langle z ; y, x\rangle=-\langle z ; x, y\rangle, \\
& \left(\mathrm{HLY}_{5}\right) \sigma\{(x \cdot y) \cdot \alpha(z)+\langle x ; y, z\rangle\}=0, \\
& \left(\mathrm{HLY}_{6}\right) \sigma\{\langle\alpha(u) ; \alpha(z), y \cdot x\rangle\}=0, \\
& \left(\mathrm{HLY}_{7}\right)\langle v \cdot u ; \alpha(y), \alpha(x)\rangle=\langle v ; y, x\rangle \cdot \alpha^{2}(u)+\alpha^{2}(v) \cdot\langle u ; y, x\rangle,
\end{aligned}
$$


$\left(\mathrm{HLY}_{8}\right)\left\langle\langle w ; v, u\rangle ; \alpha^{2}(y), \alpha^{2}(x)\right\rangle=\left\langle\langle w ; y, x\rangle ; \alpha^{2}(v), \alpha^{2}(u)\right\rangle+\left\langle\alpha^{2}(w) ;\right.$

$\left.\langle v ; y, x\rangle, \alpha^{2}(u)\right\rangle+\left\langle\alpha^{2}(w) ; \alpha^{2}(v),\langle u ; y, x\rangle\right\rangle$,

for all $u, v, w, x, y, z \in L$.

Remark 2.8. From a (left) Hom-Lie-Yamaguti algebra $(L, \star,\{, ;\}, \alpha)$, one can get a right Hom-Lie-Yamaguti algebra $(L, \cdot,\langle;\rangle,, \alpha)$, where $x \cdot y=-y \star x,\langle z ; y, x\rangle=-\{y, x ; z\}$ and conversely.

As Corollary 3.2 in [5], one can check that if $(L, \cdot,\langle;\rangle$,$) is a (right)$ Lie-Yamaguti algebra and $\alpha$ an endomorphism of $(L, \cdot,\langle;\rangle$,$) , then$ $L_{\alpha}=\left(L,{ }_{\alpha}=\alpha \circ \cdot\langle;,\rangle_{\alpha}=\alpha^{2} \circ\langle;\rangle,, \alpha\right)$ is a (right) Hom-Lie-Yamaguti algebra.

Now, let prove the following result which says that a right Hom-LieYamaguti Algebra is closed under taking $n$-th-derived binary-ternary Hom-algebras.

Theorem 2.9. Let $(L, \cdot,\langle;\rangle,, \alpha)$ be a right Hom-Lie-Yamaguti algebra. Then for each $n \in \mathbb{N}$, the $n$-th-derived Hom-algebra $L^{(n)}=\left(L,{ }^{(n)}=\right.$ $\left.\left.\alpha^{2^{n}-1} \circ \cdot\langle;\rangle,\right\rangle^{\langle n\rangle}=\alpha^{2^{n+1}-2} \circ\langle;,\rangle,, \alpha^{2^{n}}\right)$ is also a right Hom-Lie-Yamaguti algebra.

Proof. The identities $\left(\mathrm{HLY}_{1}-\mathrm{HLY}_{6}\right)$ for $L^{(n)}$ are obvious. Next, using $\left(\mathrm{HLY}_{7}\right)$ for $L$, the checking of $\left(\mathrm{HLY}_{7}\right)$ for $L^{(n)}$ is as it follows:

$$
\begin{aligned}
\left\langle v \cdot{ }^{(n)} u ;\right. & \left.\alpha^{2^{n}}(y), \alpha^{2^{n}}(x)\right\rangle^{(n)} \\
& =\alpha^{2^{n+1}-2}\left(\left\langle\alpha^{2^{n}-1}(v \cdot u) ; \alpha^{2^{n}-1} \alpha(y), \alpha^{2^{n}-1} \alpha(x)\right\rangle\right) \\
& =\alpha^{2^{n+1}-2} \alpha^{2^{n}-1}(\langle(v \cdot u) ; \alpha(y), \alpha(x)\rangle)
\end{aligned}
$$




$$
\begin{aligned}
& =\alpha^{2^{n+1}-2} \alpha^{2^{n}-1}\left(\langle v ; y, x\rangle \cdot \alpha^{2}(u)+\alpha^{2}(v) \cdot\langle u ; y, x\rangle\right) \\
& =\alpha^{2^{n+1}-2}\left(\langle v ; y, x\rangle \cdot{ }^{(n)} \alpha^{2}(u)+\alpha^{2}(v) \cdot{ }^{(n)}\langle u ; y, x\rangle\right) \\
& =\langle v ; y, x\rangle^{(n)} \cdot{ }^{(n)} \alpha^{2^{n+1}}(u)+\alpha^{2^{n+1}}(v) \cdot{ }^{(n)}\langle u ; y, x\rangle^{(n)} \\
& =\langle v ; y, x\rangle^{(n)} \cdot(n)\left(\alpha^{2^{n}}\right)^{2}(u)+\left(\alpha^{2^{n}}\right)^{2}(v) \cdot{ }^{(n)}\langle u ; y, x\rangle^{(n)},
\end{aligned}
$$

and thus $\left(\mathrm{HLY}_{7}\right)$ holds for $L^{(n)}$. Finally using the identity $\left(\mathrm{HLY}_{8}\right)$ for $L$, the verification of $\left(\mathrm{HLY}_{8}\right)$ for $L^{(n)}$ is as follows:

$$
\begin{aligned}
&\left\langle\langle w ; v, u\rangle^{(n)} ;\left(\alpha^{2^{n}}\right)^{2}(y),\left(\alpha^{2^{n}}\right)^{2}(x)\right\rangle^{(n)} \\
&= \alpha^{2^{n+1}-2}\left(\left\langle\alpha^{2^{n+1}-2}(\langle w ; v, u\rangle) ; \alpha^{2^{n+1}}(y), \alpha^{2^{n+1}}(x)\right\rangle\right) \\
&=\left(\alpha^{2^{n+1}-2}\right)^{2}\left(\left\langle\langle w ; v, u\rangle ; \alpha^{2}(y), \alpha^{2}(x)\right\rangle\right) \\
&=\left(\alpha^{2^{n+1}-2}\right)^{2}\left(\left\langle\langle w ; y, x\rangle ; \alpha^{2}(v), \alpha^{2}(u)\right\rangle\right. \\
&\left.+\left\langle\alpha^{2}(w) ;\langle v ; y, x\rangle, \alpha^{2}(u)\right\rangle+\left\langle\alpha^{2}(w) ; \alpha^{2}(v),\langle u ; y, x\rangle\right\rangle\right) \\
&=\left\langle\langle w ; y, x\rangle^{(n)} ; \alpha^{2^{n+1}}(v), \alpha^{2^{n+1}}(u)\right\rangle^{(n)} \\
&+\left\langle\alpha^{2^{n+1}}(w) ;\langle v ; y, x\rangle^{(n)},(\alpha)^{2^{n+1}}(u)\right\rangle^{(n)} \\
&+\left\langle\alpha^{2^{n+1}}(w) ; \alpha^{2^{n+1}}(v),\langle u ; y, x\rangle^{(n)}\right\rangle^{(n)} \\
&=\left\langle\langle w ; y, x\rangle^{(n)} ;\left(\alpha^{2^{n}}\right)^{2}(v),\left(\alpha^{2^{n}}\right)^{2}(u)\right\rangle^{(n)} \\
&+\left\langle\left(\alpha^{2^{n}}\right)^{2}(w) ;\langle v ; y, x\rangle^{(n)},\left(\alpha^{2^{n}}\right)^{2}(u)\right\rangle^{(n)} \\
&+\left\langle\left(\alpha^{2^{n}}\right)^{2}(w) ;\left(\alpha^{2^{n}}\right)^{2}(v),\langle u ; y, x\rangle^{(n)}\right\rangle^{(n)} .
\end{aligned}
$$

Thus $\left(\mathrm{HLY}_{8}\right)$ holds for $L^{(n)}$. Therefore, we get that $L^{(n)}$ is a right HomLie-Yamaguti algebra. 
We get the following way for constructing a right Hom-Lie-Yamaguti algebra from a left Leibniz algebra. One can straightforward check that the same result holds for a right Leibniz algebra.

Proposition 2.10. Let $(L, \cdot)$ be a left Leibniz algebra and $\beta$, an endomorphism of L. Define on L, the operations

$$
\begin{aligned}
{[x, y]_{\beta} } & =\beta(x \cdot y-y \cdot x), \\
\langle z ; x, y\rangle_{\beta} & =-\beta^{2}(x y \cdot z) .
\end{aligned}
$$

Then $\left(L,[,]_{\beta},\langle;,\rangle_{\beta}, \beta\right)$ is a right Hom-Lie-Yamaguti.

Proof. Consider on $L$, the ternary operation $\langle z ; x, y\rangle=-x y \cdot z$ and the commutator $[x, y]=x \cdot y-y \cdot x, \forall x, y, z \in L$, then $(L,[],,\langle;\rangle$,$) is a$ right Lie-Yamaguti [14] (Proposition 3.3). Moreover, since $\beta$ is an endomorphism of $L$, we have $\beta([x, y])=\beta(x \cdot y-y \cdot x)=\beta(x) \cdot \beta(y)-$ $\beta(y) \cdot \beta(x)=[\beta(x), \beta(y)]$ and $\beta(\langle z ; x, y\rangle)=\beta(-x y \cdot z)=-\beta(x) \beta(y) \cdot \beta(z)$ $=\langle\beta(z) ; \beta(x), \beta(y)\rangle$, then $\beta$ is also an endomorphism of $(L,[],,\langle,\rangle$, and therefore, as a similar way as Corollary 3.2 in $[5],\left(L,[,]_{\beta},\langle;,\rangle_{\beta}, \beta\right)$ is a right Hom-Lie-Yamaguti algebra.

We get also the following:

Theorem 2.11. Let $(A, \cdot, \alpha)$ be a Hom-Lie algebra. Then $(A, \cdot,[;],, \alpha)$ is a right Hom-Lie-Yamaguti algebra, where

$$
[z ; y, x]=x y \cdot \alpha(z)
$$

for all $x, y, z \in A$.

Proof. The proof of this theorem is to verify the identities $\left(\mathrm{HLY}_{1}\right)-\left(\mathrm{HLY}_{8}\right)$ : As $(L, \cdot, \alpha)$ is a multiplicative Hom-algebra, then the identities $\left(\mathrm{HLY}_{1}\right)$ and $\left(\mathrm{HLY}_{2}\right)$ hold for $(A, \cdot,[;],, \alpha)$. Using the skewsymmetry of the operation "." of $(A, \cdot, \alpha)$, we compute

$$
[z ; y, x]=(x \cdot y) \cdot \alpha(z)=-(y \cdot x) \cdot \alpha(z)=-[z ; x, y],
$$


and then the identities $\left(\mathrm{HLY}_{3}\right)$ and $\left(\mathrm{HLY}_{4}\right)$ hold for $(A, \cdot,[;],, \alpha)$. We compute the identity $\left(\mathrm{HLY}_{5}\right)$ as follows:

$$
\begin{aligned}
\cup_{(x, y, z)}\{(x \cdot y) \cdot \alpha(z)+[z ; y, x]\}= & \cup_{(x, y, z)}\{(x \cdot y) \cdot \alpha(z) \\
& +(y \cdot x) \cdot \alpha(z)\} \\
= & 2 \cup_{(x, y, z)}(x \cdot y) \cdot \alpha(z) \\
= & 0 \text { (by the Hom-Jacobi identity). }
\end{aligned}
$$

Consider now $\cup_{(x, y, z)}\{[\alpha(u) ; \alpha(z), y \cdot x]\}$. Then

$$
\begin{aligned}
\mho_{(x, y, z)}\{[\alpha(u) ; \alpha(z), y \cdot x]\} & =\mho_{(x, y, z)}\{(y \cdot x) \cdot \alpha(z)\} \cdot \alpha^{2}(u) \\
& =-\circlearrowleft_{(x, y, z)}\{(x \cdot y) \cdot \alpha(z)\} \cdot \alpha^{2}(u) \\
& =0 \text { (again by the Hom-Jacobi identity). }
\end{aligned}
$$

So that we get $\left(\mathrm{HLY}_{6}\right)$. Next,

$$
\begin{aligned}
U_{(x, y, z)}[v \cdot u ; \alpha(y), \alpha(x)]= & (\alpha(x) \cdot \alpha(y)) \cdot \alpha(v \cdot u) \\
= & \alpha(x \cdot y) \cdot(\alpha(v) \cdot \alpha(u)) \text { (by multiplicativity) } \\
= & -\alpha^{2}(v) \cdot(\alpha(u) \cdot(x \cdot y))-\alpha^{2}(u) \cdot((x \cdot y) \cdot \alpha(v)) \\
& \quad(\text { by the Hom-Jacobi identity) } \\
= & \alpha^{2}(v) \cdot((x \cdot y) \cdot \alpha(u))+((x \cdot y) \cdot \alpha(v)) \cdot \alpha^{2}(u) \\
& \quad(\text { by skew-symmetry) } \\
= & {\left.[v ; y, x)] \cdot \alpha^{2}(u)+\alpha^{2}(v) \cdot[u ; y, x)\right] \text { (by (4)) } }
\end{aligned}
$$


which is $\left(\mathrm{HLY}_{7}\right)$. Finally, the identity $\left(\mathrm{HLY}_{8}\right)$ is computed as follows:

$\left[[w ; y, x] ; \alpha^{2}(v), \alpha^{2}(u)\right]+\left[\alpha^{2}(w) ;[v ; y, x], \alpha^{2}(u)\right]+\left[\alpha^{2}(w) ; \alpha^{2}(v),[u ; y, x]\right]$

$$
\begin{aligned}
= & \alpha^{2}(u) \alpha^{2}(v) \cdot \alpha(x y \cdot \alpha(w))+\alpha^{2}(u)(x y \cdot \alpha(v)) \cdot \alpha^{3}(w) \\
& +(x y \cdot \alpha(u)) \alpha^{2}(v) \cdot \alpha^{3}(w) \\
= & \alpha^{2}(u) \alpha^{2}(v) \cdot \alpha(x y \cdot \alpha(w))-\alpha^{3}(w) \cdot[\alpha(\alpha(u)) \cdot(x y \cdot \alpha(v)) \\
& +\alpha(\alpha(v)) \cdot(\alpha(u) \cdot x y)] \text { (by skew-symmetry) } \\
= & \alpha^{2}(u) \alpha^{2}(v) \cdot \alpha(x y \cdot \alpha(w))+\alpha^{3}(w) \cdot(\alpha(x y) \cdot \alpha(v) \alpha(u))
\end{aligned}
$$

(by Hom-Jacobi identity)

$=\alpha^{2}(u v) \cdot \alpha(x y) \alpha^{2}(w)+\alpha^{3}(w) \cdot \alpha(x y) \alpha(v u)$ (by multiplicativity)

$=\alpha(\alpha(u v)) \cdot \alpha(x y) \alpha^{2}(w)+\alpha\left(\alpha^{2}(w)\right) \cdot \alpha(u v) \alpha(x y)$ (by skew-symmetry)

$=-\alpha^{2}(x y) \cdot \alpha^{2}(w) \alpha(u v)$ (by Hom-Jacobi identity)

$=\alpha^{2}(x) \alpha^{2}(y) \cdot \alpha(u v \cdot \alpha(w))$ (by skew-symmetry and multiplicativity)

$=\left[[w ; v, u] ; \alpha^{2}(y), \alpha^{2}(x)\right]$ (by (4)).

Therefore $(A, \cdot,[;],, \alpha)$ is a right Hom-Lie-Yamaguti algebra.

Example 2.12. From the family of three-dimensional complex HomLie algebras $\operatorname{sl}(2, \mathbb{C})_{\lambda}=\left(\operatorname{sl}(2, \mathbb{C}),[,]_{\alpha_{\lambda}}, \alpha_{\lambda}\right)(\lambda \neq 0)$ with a basis $(e, f, h)$ where $e=\left(\begin{array}{ll}0 & 1 \\ 0 & 0\end{array}\right), f=\left(\begin{array}{ll}0 & 0 \\ 1 & 0\end{array}\right)$, and $h=\left(\begin{array}{cc}1 & 0 \\ 0 & -1\end{array}\right)$ of Example 2.4, we get thanks to Theorem 2.11, the family of three-dimensional complex right Hom-Lie-Yamaguti algebras $\operatorname{HLY}(\operatorname{sl}(2, \mathbb{C}))_{\lambda}=(\operatorname{sl}(2, \mathbb{C})$, $\left.[,]_{\alpha_{\lambda}},[;,,]_{\alpha_{\lambda}}, \alpha_{\lambda}\right)(\lambda \neq 0)$ defined by $\alpha_{\lambda}(e)=\lambda e, \alpha_{\lambda}(f)=\frac{1}{\lambda} f, \alpha_{\lambda}(h)=h$, 
$[e, f]_{\lambda}=h,[h, f]_{\lambda}=-\frac{2}{\lambda} f,[h, e]_{\lambda}=2 \lambda e$ and $[e ; f, h]_{\alpha \lambda}=2 h,[f ; h, e]_{\alpha \lambda}$ $=-2 h,[e ; e, f]_{\alpha_{\lambda}}=-2 \lambda^{2} e,[f ; f, e]_{\alpha_{\lambda}}=-\frac{2}{\lambda^{2}},[h ; h, e]_{\alpha_{\lambda}}=4 \lambda^{2} e,[h ; h, f]_{\alpha \lambda}$ $=\frac{4}{\lambda^{2}}$.

We think of the collection $\left\{H L Y\left((\operatorname{sl}(2, \mathbb{C}))_{\lambda}: \lambda \in \mathbb{C} \backslash\{0\}\right\}\right.$ as a oneparameter family of deformations of $\operatorname{sl}(2, \mathbb{C})$ into Hom-Lie Yamaguti algebras.

Left Hom-Bol algebras are studied in [4] under the name Hom-Bol algebra. Here, we consider the right one and the reader is advised not to confuse it with the one of Hom-Bol algebra.

Definition 2.13. A right Hom-Bol algebra is a quadruple $(B, *,\langle;\rangle,, \alpha)$ in which $B$ is $\mathbb{K}$-vector space, “*” a binary operation, " $\langle;$,$\rangle " ternary$ operation on $B$ and $\alpha: B \rightarrow B$ a linear map such that

$$
\begin{aligned}
& \left(\mathrm{HB}_{1}\right) \alpha(x * y)=\alpha(x) * \alpha(y), \\
& \left(\mathrm{HB}_{2}\right) \alpha(\langle x ; y, z\rangle)=\langle\alpha(x) ; \alpha(y), \alpha(z)\rangle, \\
& \left(\mathrm{HB}_{3}\right) x * y=-y * x, \\
& \left(\mathrm{HB}_{4}\right)\langle z ; y, x\rangle=-\langle z ; x, y\rangle, \\
& \left(\mathrm{HB}_{5}\right) \sigma\langle z ; y, x\rangle=0, \\
& \left(\mathrm{HB}_{6}\right)\langle v * u ; \alpha(y), \alpha(x)\rangle=\langle v ; y, x\rangle * \alpha^{2}(u)+\alpha^{2}(v) *\langle u ; y, x\rangle \\
& \quad-(\alpha(y) * \alpha(x)) *(\alpha(v) * \alpha(u)), \\
& \left(\mathrm{HB}_{7}\right)\left\langle\langle w ; v, u\rangle ; \alpha^{2}(y), \alpha^{2}(x)\right\rangle=\left\langle\langle w ; y, x\rangle ; \alpha^{2}(v), \alpha^{2}(u)\right\rangle \\
& \quad+\left\langle\alpha^{2}(w) ;\langle v ; y, x\rangle, \alpha^{2}(u)\right\rangle \\
& \quad+\left\langle\alpha^{2}(w) ; \alpha^{2}(v),\langle u ; y, x\rangle\right\rangle,
\end{aligned}
$$

for all $u, v, w, x, y, z \in B$. 
Remark 2.14. From a left Hom-Bol algebra $(B, \cdot,\{, ;\}, \alpha)$ one gets a right Hom-Bol $(B, *,\langle;\rangle,, \alpha)$, where $x * y=-y \cdot x,\langle z ; y, x\rangle=-\{y, x ; z\}$ and conversely.

Theorem 2.15. Let $\left(A_{l}, \cdot\right)$ be a left alternative algebra and $\beta$ an endomorphism of $A_{l}$. Define on $A_{l}$ the operations [, ]' and $\langle;,\rangle^{\prime}$ by

$$
\begin{aligned}
{[x, y]^{\prime} } & :=\beta(x \cdot y-y \cdot x), \\
\langle z ; y, x\rangle^{\prime} & :=\beta^{2}(x \cdot y z-y \cdot x z-z \cdot[x, y]),
\end{aligned}
$$

for all $x, y, z \in A_{l}$, then $\left(A_{l},[,]^{\prime},\langle;,\rangle^{\prime}, \beta\right)$ is a right Hom-Bol algebra.

Proof. Consider on $A_{l}$, the commutator $[x, y]:=x \cdot y-y \cdot x$ and the ternary operation $\langle z ; y, x\rangle=x \cdot y z-y \cdot x z-z \cdot[x, y], \quad \forall x, y, z \in A_{l}$, then similarly to $[8,20]$, one can check that $\left(A_{l},[],,\langle;\rangle,\right)$ is a right Bol algebra. Moreover, since $\beta$ is an endomorphism of $\left(A_{l}, \cdot\right)$, we have $\beta([x, y])=\beta(x) \cdot \beta(y)-\beta(y) \cdot \beta(x)=[\beta(x), \beta(y)]$ and $\beta(\langle z ; y, x\rangle)=\beta(x) \cdot \beta$ $(y) \beta(z)-\beta(y) \cdot \beta(x) \beta(z)-\beta(z) \cdot[\beta(x), \beta(y)]=\langle\beta(z) ; \beta(y), \beta(x)\rangle$, then $\beta$ is also an endomorphism of $\left(A_{l},[],,\langle;\rangle,\right)$. Therefore, similarly to Corollary 3.3 in [4] for right $\mathrm{Bol}$ algebra implies that $\left(A_{l},[,]^{\prime},\langle;,\rangle^{\prime}, \beta\right)$ is right Hom-Bol algebra.

Example 2.16. Consider one of the 4-dimensional left alternative (but not associative) algebra $\left(A_{l}, \cdot\right)$ over any field. With respect to a basis $\left(e_{0}, e_{1}, e_{2}, e_{3}\right)$, the non-zero products are defined by $e_{0} \cdot e_{0}=e_{0}$, $e_{0} \cdot e_{1}=e_{1}, e_{2} \cdot e_{0}=e_{2}, e_{2} \cdot e_{3}=e_{1}, e_{3} \cdot e_{0}=e_{3}, e_{3} \cdot e_{2}=-e_{1}$ (see [6] p. 144). It is easy to check that the linear map $\beta$ defined by $\beta\left(e_{0}\right)=e_{0}+e_{2}$, $\beta\left(e_{1}\right)=0, \beta\left(e_{2}\right)=2 e_{2}, \beta\left(e_{3}\right)=e_{2}$ is a morphism of $\left(A_{l}, \cdot\right)$. Thus, thank to Theorem 2.15, $\left(A_{l},[,]^{\prime},\langle,,\rangle^{\prime}, \beta\right)$ is right Hom-Bol algebra where the nonzero products are given by $\left[e_{0}, e_{2}\right]^{\prime}=-2 e_{2},\left[e_{0}, e_{3}\right]^{\prime}=-e_{2}$ and $\left\langle e_{0} ; e_{2}, e_{0}\right\rangle^{\prime}=-4 e_{2},\left\langle e_{0} ; e_{3}, e_{0}\right\rangle^{\prime}=-2 e$. 
Remark 2.17. We get the similar result for right alternative algebras.

\section{Definition and Construction Theorems}

In this section, we define a Hom-hyporeductive triple algebra. It turns out that Hom-hyporeductive triple algebras constitute a twisted generalization of hyporeductive triple algebras. We prove some construction theorems for Hom-hyporeductive triple algebras (Theorem 3.3, Theorm 3.6, Corollary 3.4, Corollary 3.5). Theorem 3.3 shows that the category of Hom-hyporeductive triple algebras is closed under selfmorphisms while Corollary 3.5 says that every hyporeductive triple algebra can be twisted, along any endomorphism, into a Homhyporeductive triple algebra. Theorem 3.6 points out that Homhyporeductive triple algebras are also closed under taking derived binaryternary Hom-algebras. We begin with the definition of the basic object of this paper.

Definition 3.1. A Hom-hyporeductive triple algebra is a quintuple $(V, \cdot, *,\langle;\rangle,, \alpha)$ consisting of a vector space $V$, bilinear maps , $*: V \times V \rightarrow V$, a trilinear map $\langle;\rangle:, V \times V \times V \rightarrow V$ and a linear map $\alpha: V \rightarrow V$ such that

$$
\begin{aligned}
& \left(\mathrm{HH}_{1}\right) x \cdot y=-y \cdot x, \\
& \left(\mathrm{HH}_{2}\right) x * y=-y * x, \\
& \left(\mathrm{HH}_{3}\right)\langle z ; y, x\rangle=-\langle z ; x, y\rangle, \\
& \left(\mathrm{HH}_{4}\right) \alpha(x \cdot y)=\alpha(x) \cdot \alpha(y), \\
& \left(\mathrm{HH}_{5}\right) \alpha(x * y)=\alpha(x) * \alpha(y), \\
& \left(\mathrm{HH}_{6}\right) \alpha(\langle z ; y, x\rangle)=\langle\alpha(z) ; \alpha(y), \alpha(x)\rangle,
\end{aligned}
$$


$\left(\mathrm{HH}_{7}\right) \sigma\{\alpha(x) \cdot(y \cdot z)-\langle x ; y, z\rangle\}=0$,

$\left(\mathrm{HH}_{8}\right) \sigma\{\alpha(z) *(x \cdot y)\}=0$,

$\left(\mathrm{HH}_{9}\right) \sigma\langle\alpha(t) ; \alpha(z), x \cdot y\rangle=0$,

$\left(\mathrm{HH}_{10}\right) \alpha^{2}(x) \cdot\langle y ; z, t\rangle-\alpha^{2}(y) \cdot\langle x ; z, t\rangle+\langle y \cdot x ; \alpha(z), \alpha(t)\rangle$

$$
\begin{aligned}
& -\langle z * t ; \alpha(y), \alpha(x)\rangle+\langle y * x ; \alpha(z), \alpha(t)\rangle-\alpha^{2}(y) *\langle x ; z, t\rangle \\
& +\alpha^{2}(x) *\langle y ; z, t\rangle-(\alpha(z) * \alpha(t)) *(\alpha(y) * \alpha(x)) \\
& -(\alpha(z) * \alpha(t)) \cdot(\alpha(y) * \alpha(x))=0
\end{aligned}
$$

$\left(\mathrm{HH}_{11}\right) \alpha^{3}(u) \cdot\left(\alpha^{2}(x) \cdot\langle y ; z, t\rangle-\alpha^{2}(y) \cdot\langle x ; z, t\rangle+\langle y \cdot x ; \alpha(z), \alpha(t)\rangle\right)$

$$
\begin{aligned}
& +\left\langle\langle u ; z, t\rangle ; \alpha^{2}(y), \alpha^{2}(x)\right\rangle-\left\langle\langle u ; y, x\rangle ; \alpha^{2}(z), \alpha^{2}(t)\right\rangle \\
& +\left\langle\alpha^{2}(u) ; \alpha^{2}(y),\langle x ; z, t\rangle\right\rangle-\left\langle\alpha^{2}(u) ; \alpha^{2}(x),\langle y ; z, t\rangle\right\rangle=0,
\end{aligned}
$$

$\left(\mathrm{HH}_{12}\right) \alpha^{3}(u) *\left(\alpha^{2}(x) \cdot\langle y ; z, t\rangle-\alpha^{2}(y) \cdot\langle x ; z, t\rangle+\langle y \cdot x ; \alpha(z), \alpha(t)\rangle\right)=0$,

$\left(\mathrm{HH}_{13}\right)\left\langle\alpha^{3}(v) ; \alpha^{3}(u), \alpha^{2}(x) \cdot\langle y ; z, t\rangle-\alpha^{2}(y) \cdot\langle x ; z, t\rangle\right.$

$$
+\langle y \cdot x ; \alpha(z), \alpha(t)\rangle\rangle=0
$$

$\left(\mathrm{HH}_{14}\right) \alpha^{2}(x) \cdot\langle y ; z, t\rangle-\alpha^{2}(y) \cdot\langle x ; z, t\rangle+\langle y \cdot x ; \alpha(z), \alpha(t)\rangle$

$$
+\alpha^{2}(t) \cdot\langle z ; y, x\rangle-\alpha^{2}(z) \cdot\langle t ; y, x\rangle+\langle z \cdot t ; \alpha(y), \alpha(x)\rangle=0
$$

$\left(\mathrm{HH}_{15}\right) \alpha^{2}(y) *\langle x ; z, t\rangle-\alpha^{2}(x) *\langle y ; z, t\rangle+\alpha^{2}(z) *\langle t ; y, x\rangle$

$$
-\alpha^{2}(t) *\langle z ; y, x\rangle=0,
$$




$$
\begin{aligned}
\left(\mathrm{HH}_{16}\right) & \sum\left\{\left\langle\left(\langle z \cdot t ; \alpha(y), \alpha(x)\rangle+\alpha^{2}(t) \cdot\langle z ; y, x\rangle-\alpha^{2}(z) \cdot\langle t ; y, x\rangle\right) ;\right.\right. \\
& \left.\alpha^{3}(u), \alpha^{3}(v)\right\rangle+\left\langle\alpha^{2}(u) \cdot \alpha^{2}(v) ;\langle\alpha(t) ; \alpha(y), \alpha(x)\rangle, \alpha^{3}(z)\right\rangle \\
+ & \alpha^{4}(v) \cdot\left\langle\alpha^{2}(u) ;\langle t ; y, x\rangle, \alpha^{2}(z)\right\rangle-\alpha^{4}(u) \cdot\left\langle\alpha^{2}(v) ;\langle t ; y, x\rangle, \alpha^{2}(z)\right\rangle \\
& \left(\left\langle\alpha^{2}(u) \cdot \alpha^{2}(v) ;\langle\alpha(z) ; \alpha(y), \alpha(x)\rangle, \alpha^{3}(t)\right\rangle+\alpha^{4}(v) \cdot\left\langle\alpha^{2}(u) ;\right.\right. \\
& \left.\left.\left.\langle z ; y, x\rangle, \alpha^{2}(t)\right\rangle-\alpha^{4}(u) \cdot\left\langle\alpha^{2}(v) ;\langle z ; y, x\rangle, \alpha^{2}(t)\right\rangle\right)\right\}=0, \\
\left(\mathrm{HH}_{17}\right) & \sum\left\{\left(\left\langle\alpha^{2}(v) ;\langle t ; y, x\rangle, \alpha^{2}(z)\right\rangle-\left\langle\alpha^{2}(v) ;\langle z ; y, x\rangle, \alpha^{2}(t)\right\rangle\right) * \alpha^{4}(u)\right. \\
+ & \left.\left(\left\langle\alpha^{2}(u) ;\langle z ; y, x\rangle, \alpha^{2}(t)\right\rangle-\left\langle\alpha^{2}(u) ;\langle t ; y, x\rangle, \alpha(z)\right\rangle\right) * \alpha^{4}(v)\right\}=0, \\
\left(\mathrm{HH}_{18}\right) & \sum\left\{\left\langle\alpha^{4}(w) ;\left(\left\langle\alpha^{2}(v) ;\langle t ; y, x\rangle, \alpha^{2}(z)\right\rangle-\left\langle\alpha^{2}(v) ;\langle z ; y, x\rangle, \alpha^{2}\right.\right.\right.\right. \\
& \left.(t)\rangle), \alpha^{4}(u)\right\rangle+\left\langle\alpha^{4}(w) ;\left(\left\langle\alpha^{2}(u) ;\langle z ; y, x\rangle, \alpha^{2}(t)\right\rangle-\left\langle\alpha^{2}(u) ;\right.\right.\right. \\
& \left.\left.\left.\left.\langle t ; y, x\rangle, \alpha^{2}(z)\right\rangle\right), \alpha^{4}(v)\right\rangle\right\}=0,
\end{aligned}
$$

where $\sigma$ denotes the sum over cyclic permutation of $x, y, z$ and $\sum$ is the one over $(z, t),(y, x)$ and $(u, v)$ for all $x, y, z, t, v, w \in V$.

Remark 3.2. (i) If $\alpha=I d$, then the Hom-hyporeductive triple algebra $(V, \cdot, *,\langle;\rangle,, \alpha)$ reduces to the hyporeductive triple algebra $(V, \cdot, *,\langle;\rangle$,$) . So a hyporeductive triple algebra may be seen as a Hom-$ hyporeductive triple algebras with the identity map $I d$ as the twisting map.

(ii) If $x * y=0$, for all $x, y \in A$, then $(V, \cdot, *,\langle;\rangle,, \alpha)$ becomes a right Hom-Lie-Yamaguti algebra $(V, \cdot,\langle;\rangle,, \alpha)$ (see Definition 2.7).

(iii) If $x \cdot y=0$, for all $x, y \in A$ then $(V, \cdot, *,\langle;\rangle,, \alpha)$ becomes a right Hom-Bol algebra $(V, *,\langle;\rangle,, \alpha)$ (see Definition 2.13). 
The following result produces a sequence of Hom-hyporeductive triple algebras. It says again that Hom-hyporeductive triple algebras are closed by self-morphisms.

Theorem 3.3. Let $(V, \cdot, \cdot,\langle;\rangle,, \alpha)$ be a Hom-hyporeductive triple algebra and $\beta$ be a morphism of $(V, \cdot, \cdot,\langle;\rangle,, \alpha)$. Let $\beta^{0}=I d_{V}$ and for $n \geq 0, \beta^{n}:=\beta \beta^{n-1}\left(=\beta \circ \beta^{n-1}\right)$. Define on $V$ the operations: $x \cdot \beta^{n} y:=$ $\beta^{n}(x \cdot y), x *_{\beta^{n}} y:=\beta^{n}(x * y) \quad$ and $\quad\langle x ; y, z\rangle_{\beta^{n}}:=\beta^{2 n}(\langle x ; y, z\rangle)$, for all $x, y, z \in V$. Then $V_{\beta^{n}}:=\left(V,{ }_{\beta^{n}}, *_{\beta^{n}},\langle;,\rangle_{\beta^{n}}, \beta^{n} \alpha\right)$ is a Hom-hyporeductive triple algebra.

Proof. We observe that the skew-symmetry identities $\left(\mathrm{HH}_{1}\right),\left(\mathrm{HH}_{2}\right)$, and $\left(\mathrm{HH}_{3}\right)$ for $V_{\beta^{n}}$ follow from the skew-symmetry of *, and $\langle;$,$\rangle ,$ respectively. The condition $\beta \alpha=\alpha \beta$ implies $\beta^{n} \alpha=\alpha \beta^{n}$ which with the definition of ${ }_{\beta^{n}}, *_{\beta^{n}}$ and $\langle;,\rangle_{\beta^{n}}$ lead to the condition $\left(\mathrm{HH}_{4}\right)$ to $\left(\mathrm{HH}_{6}\right)$. Next using the condition $\alpha \beta=\beta \alpha$, we have

$$
\begin{aligned}
\sigma\left\{\beta^{n} \alpha(x) \cdot \beta^{n}\left(y \cdot \beta^{n} z\right)-\langle x ; y, z\rangle_{\beta^{n}}\right\} \\
=\sigma\left\{\beta^{n}\left(\beta^{n} \alpha(x) \cdot\left(\beta^{n}(y \cdot z)\right)\right)-\beta^{2 n}(\langle x ; y, z\rangle)\right\} \\
=\sigma\left\{\beta^{2 n}(\alpha(x) \cdot(y \cdot z))-\beta^{2 n}(\langle x ; y, z\rangle)\right\} \\
=\beta^{2 n}(\sigma\{\alpha(x) \cdot(y \cdot z)-\langle x ; y, z\rangle\}) \\
=0 \text { by }\left(\mathrm{HH}_{7}\right) \text { in } V_{\alpha} .
\end{aligned}
$$


Then we get $\left(\mathrm{HH}_{7}\right)$ for $V_{\beta^{n}}$, similarly we get $\left(\mathrm{HH}_{8}\right)$ and $\left(\mathrm{HH}_{9}\right)$ for $V_{\beta^{n}}$. Using repeatedly the condition $\alpha \beta=\beta \alpha$, we prove $\left(\mathrm{HH}_{10}\right)$ to $\left(\mathrm{HH}_{15}\right)$ as it follows:

$$
\begin{aligned}
& \left(\beta^{n} \alpha\right)^{2}(x) \cdot{ }_{\beta^{n}}\langle y ; z, t\rangle_{\beta^{n}}-\left(\beta^{n} \alpha\right)^{2}(y) \cdot{ }_{\beta^{n}}\langle x ; z, t\rangle_{\beta^{n}} \\
& +\left\langle y \cdot{ }_{\beta^{n}} x ; \beta^{n} \alpha(z), \beta^{n} \alpha(t)\right\rangle_{\beta^{n}}-\left\langle z *_{\beta^{n}} t ; \beta^{n} \alpha(y), \beta^{n} \alpha(x)\right\rangle_{\beta^{n}} \\
& +\left\langle y *_{\beta^{n}} x ; \beta^{n} \alpha(z), \beta^{n} \alpha(t)\right\rangle_{\beta^{n}}-\left(\beta^{n} \alpha\right)^{2}(y) *_{\beta^{n}}\langle x ; z, t\rangle_{\beta^{n}} \\
& +\left(\beta^{n} \alpha\right)^{2}(x) *_{\beta^{n}}\langle y ; z, t\rangle_{\beta^{n}}-\left(\beta^{n} \alpha(z) *_{\beta^{n}} \beta^{n} \alpha(t)\right) *_{\beta^{n}}\left(\beta^{n} \alpha(y) *_{\beta^{n}} \beta^{n} \alpha(x)\right) \\
& -\left(\beta^{n} \alpha(z) *_{\beta^{n}} \beta^{n} \alpha(t)\right) \cdot \beta^{n}\left(\beta^{n} \alpha(y) *_{\beta^{n}} \beta^{n} \alpha(x)\right) \\
& =\beta^{n}\left(\beta^{2 n} \alpha^{2}(x) \cdot \beta^{2 n}\langle y ; z, t\rangle\right)-\beta^{n}\left(\beta^{2 n} \alpha^{2}(y) \cdot \beta^{2 n}(\langle x ; z, t\rangle)\right) \\
& +\beta^{2 n}\left(\left\langle\beta^{n}(y \cdot x) ; \beta^{n} \alpha(z), \beta^{n} \alpha(t)\right\rangle\right)-\beta^{2 n}\left(\left\langle\beta^{n}(z * t) ; \beta^{n} \alpha(y), \beta^{n} \alpha(x)\right\rangle\right) \\
& +\beta^{2 n}\left(\left\langle\beta^{n}(y * x) ; \beta^{n} \alpha(z), \beta^{n} \alpha(t)\right\rangle\right)-\beta^{n}\left(\beta^{2 n} \alpha^{2}(y) * \beta^{2 n}(\langle x ; z, t\rangle)\right) \\
& +\beta^{n}\left(\beta^{2 n} \alpha^{2}(x) * \beta^{2 n}(\langle y ; z, t\rangle)\right)-\beta^{n}\left(\beta^{n}\left(\beta^{n} \alpha(z) * \beta^{n} \alpha(t)\right)\right. \\
& \left.* \beta^{n}\left(\beta^{n} \alpha(y) * \beta^{n} \alpha(x)\right)\right) \\
& -\beta^{n}\left(\beta^{n}\left(\beta^{n} \alpha(z) * \beta^{n} \alpha(t)\right) \cdot \beta^{n}\left(\beta^{n} \alpha(y) * \beta^{n} \alpha(x)\right)\right) \\
& =\beta^{3 n}\left(\alpha^{2}(x) \cdot\langle y ; z, t\rangle-\alpha^{2}(y) \cdot\langle x ; z, t\rangle+\langle y \cdot x ; \alpha(z), \alpha(t)\rangle\right. \\
& -\langle z * t ; \alpha(y), \alpha(x)\rangle+\langle y * x ; \alpha(z), \alpha(t)\rangle-\alpha^{2}(y) *\langle x ; z, t\rangle \\
& +\alpha^{2}(x) *\langle y ; z, t\rangle-(\alpha(z) * \alpha(t)) *(\alpha(y) * \alpha(x)) \\
& -(\alpha(z) * \alpha(t)) \cdot(\alpha(y) * \alpha(x))) \\
& =0 \text { by }\left(\mathrm{HH}_{10}\right) \text { for } V_{\alpha} \text {; }
\end{aligned}
$$




$$
\begin{aligned}
& \left(\beta^{n} \alpha\right)^{3}(u) \cdot{ }_{\beta^{n}}\left(\left(\beta^{n} \alpha\right)^{2}(x) \cdot{ }_{\beta^{n}}\langle y ; z, t\rangle_{\beta^{n}}-\left(\beta^{n} \alpha\right)^{2}(y) \cdot{ }_{\beta^{n}}\langle x ; z, t\rangle_{\beta^{n}}\right. \\
& \left.+\left\langle y \cdot{ }_{\beta^{n}} x ; \beta^{n} \alpha(z), \beta^{n} \alpha(t)\right\rangle_{\beta^{n}}\right)+\left\langle\langle u ; z, t\rangle_{\beta^{n}} ;\left(\beta^{n} \alpha\right)^{2}(y),\left(\beta^{n} \alpha\right)^{2}(x)\right\rangle_{\beta^{n}} \\
& -\left\langle\langle u ; y, x\rangle_{\beta^{n}} ;\left(\beta^{n} \alpha\right)^{2}(z),\left(\beta^{n} \alpha\right)^{2}(t)\right\rangle_{\beta^{n}} \\
& +\left\langle\left(\beta^{n} \alpha\right)^{2}(u) ;\left(\beta^{n} \alpha\right)^{2}(y),\langle x ; z, t\rangle_{\beta^{n}}\right\rangle_{\beta^{n}} \\
& -\left\langle\left(\beta^{n} \alpha\right)^{2}(u) ;\left(\beta^{n} \alpha\right)^{2}(x),\langle y ; z, t\rangle_{\beta^{n}}\right\rangle_{\beta^{n}} \\
& =\beta^{n}\left(\beta ^ { 3 n } \alpha ^ { 3 } ( u ) \cdot \left(\beta^{n}\left(\beta^{2 n} \alpha^{2}(x) \cdot \beta^{2 n}(\langle y ; z, t\rangle)\right)-\beta^{n}\left(\beta^{2} \alpha^{2}(y) \cdot \beta^{2 n}(\langle x ; z, t\rangle)\right)\right.\right. \\
& \left.+\beta^{2 n}\left(\left\langle\beta^{n}(y \cdot x) ; \beta^{n} \alpha(z), \beta^{n} \alpha(t)\right\rangle\right)\right) \\
& +\beta^{2 n}\left(\left\langle\beta^{2 n}(\langle u ; z, t\rangle) ; \beta^{2 n} \alpha^{2}(y), \beta^{2 n} \alpha^{2}(x)\right\rangle\right) \\
& -\beta^{2 n}\left(\left\langle\beta^{2 n}(\langle u ; y, x\rangle) ; \beta^{2 n} \alpha^{2}(z), \beta^{2 n} \alpha^{2}(t)\right\rangle\right) \\
& +\beta^{2 n}\left(\left\langle\beta^{2 n} \alpha^{2}(u) ; \beta^{2 n} \alpha^{2}(y), \beta^{2 n}(\langle x ; z, t\rangle)\right\rangle\right) \\
& -\beta^{2 n}\left(\left\langle\beta^{2 n} \alpha^{2}(u) ; \beta^{2 n} \alpha^{2}(x), \beta^{2 n}(\langle y ; z, t\rangle)\right\rangle\right) \\
& =\beta^{4 n}\left(\alpha ^ { 3 } ( u ) \cdot \left(\alpha^{2}(x) \cdot\langle y ; z, t\rangle-\alpha^{2}(y) \cdot\langle x ; z, t\rangle+\langle y \cdot x ; \alpha(z), \alpha(t)\rangle\right.\right. \\
& \left.+\left\langle\langle u ; z, t\rangle ; \alpha^{2}(y), \alpha^{2}(x)\right\rangle-\langle\langle u ; y, x\rangle) ; \alpha^{2}(z), \alpha^{2}(t)\right\rangle \\
& \left.+\left\langle\alpha^{2}(u) ; \alpha^{2}(y),\langle x ; z, t\rangle\right\rangle-\left\langle\alpha^{2}(u) ; \alpha^{2}(x),\langle y ; z, t\rangle\right\rangle\right) \\
& =0 \text { by }\left(\mathrm{HH}_{11}\right) \text { for } V_{\alpha} \text {; }
\end{aligned}
$$




$$
\begin{aligned}
& \left(\beta^{n} \alpha\right)^{3}(u) *_{\beta^{n}}\left(\left(\beta^{n} \alpha\right)^{2}(x) \cdot \beta^{n}\langle y ; z, t\rangle_{\beta^{n}}-\left(\beta^{n} \alpha\right)^{2}(y) \cdot \beta^{n}\langle x ; z, t\rangle_{\beta^{n}}\right. \\
& \left.+\left\langle y \cdot \beta^{n x} ; \beta^{n} \alpha(z), \beta^{n} \alpha(t)\right\rangle_{\beta^{n}}\right) \\
& =\beta^{n}\left(\beta ^ { 3 n } \alpha ^ { 3 } ( u ) * \left(\beta^{n}\left(\beta^{2 n} \alpha^{2}(x) \cdot \beta^{2 n}(\langle y ; z, t\rangle)\right)\right.\right. \\
& \left.-\beta^{n}\left(\beta^{2 n} \alpha^{2}(y) \cdot \beta^{2 n}(\langle x ; z, t\rangle)\right)+\beta^{2 n}\left(\left\langle\beta^{n}(y \cdot x) ; \beta^{n} \alpha(z), \beta^{n} \alpha(t)\right\rangle\right)\right) \\
& =\beta^{n}\left(\beta ^ { 3 n } \alpha ^ { 3 } ( u ) * \left(\beta^{3 n} \alpha^{2}(x) \cdot \beta^{3 n}(\langle y ; z, t\rangle)-\beta^{3 n} \alpha^{2}(y) \cdot \beta^{3 n}(\langle x ; z, t\rangle)\right.\right. \\
& \left.+\beta^{3 n}(\langle y \cdot x ; \alpha(z), \alpha(t)\rangle)\right) \\
& =\beta^{4 n}\left(\alpha^{3}(u) *\left(\alpha^{2}(x) \cdot\langle y ; z, t\rangle-\alpha^{2}(y) \cdot\langle x ; z, t\rangle+\langle y \cdot x ; \alpha(z), \alpha(t)\rangle\right)\right) \\
& =0 \text { by }\left(\mathrm{HH}_{12}\right) \text { for } V_{\alpha} \text {; } \\
& \left\langle\left(\beta^{n} \alpha\right)^{3}(v) ;\left(\beta^{n} \alpha\right)^{3}(u),\left(\beta^{n} \alpha\right)^{2}(x) \cdot \beta^{n}\langle y ; z, t\rangle_{\beta^{n}}\right. \\
& \left.-\left(\beta^{n} \alpha\right)^{2}(y) \cdot \beta^{n}\langle x ; z, t\rangle_{\beta^{n}}+\left\langle y \cdot \beta^{n} x ; \beta^{n} \alpha(z), \beta^{n} \alpha(t)\right\rangle_{\beta^{n}}\right\rangle_{\beta^{n}} \\
& =\beta^{n}\left(\left\langle\beta^{3 n} \alpha^{3}(v) ; \beta^{3 n} \alpha^{3}(u), \beta^{n}\left(\beta^{2 n} \alpha^{2}(x) \cdot \beta^{2 n}(\langle y ; z, t\rangle)\right)\right.\right. \\
& \left.-\beta^{n}\left(\beta^{2 n} \alpha^{2}(y) \cdot \beta^{2 n}(\langle x ; z, t\rangle)+\beta^{2 n}\left(\left\langle\beta^{n}(y \cdot x) ; \beta^{n} \alpha(z), \beta^{n} \alpha(t)\right\rangle\right)\right\rangle\right) \\
& =\beta^{n}\left(\left\langle\beta^{3 n} \alpha^{3}(v) ; \beta^{3 n} \alpha^{2}(u), \beta^{3 n}\left(\alpha^{2}(x) \cdot\langle y ; z, t\rangle\right)-\beta^{3 n}\left(\alpha^{2}(y) \cdot\langle x ; z, t\rangle\right)\right.\right. \\
& \left.\left.+\beta^{3 n}(\langle y \cdot x ; \alpha(z), \alpha(t)\rangle)\right\rangle\right) \\
& =\beta^{4 n}\left(\left\langle\alpha^{3}(v) ; \alpha^{3}(u), \alpha^{2}(x) \cdot\langle y ; z, t\rangle-\alpha^{2}(y) \cdot\langle x ; z, t\rangle\right)\right. \\
& +\langle y \cdot x ; \alpha(z), \alpha(t)\rangle\rangle) \\
& =0 \text { by }\left(\mathrm{HH}_{13}\right) \text { for } V_{\alpha} \text {; }
\end{aligned}
$$




$$
\begin{aligned}
& \left(\beta^{n} \alpha\right)^{2}(x) \cdot{ }_{\beta^{n}}\langle y ; z, t\rangle_{\beta^{n}}-\left(\beta^{n} \alpha\right)^{2}(y) \cdot{ }_{\beta^{n}}\langle x ; z, t\rangle_{\beta^{n}} \\
& +\left\langle y \cdot{ }_{\beta^{n}} x ; \alpha(z), \alpha(t)\right\rangle_{\beta^{n}}+\left(\beta^{n} \alpha\right)^{2}(t){ }_{\beta^{n}}\langle z ; y, x\rangle_{\beta^{n}} \\
& -\left(\beta^{n} \alpha\right)^{2}(z) \cdot{ }_{\beta^{n}}\langle t ; y, x\rangle_{\beta^{n}}+\left\langle z \cdot{ }_{\beta^{n}} t ; \beta^{n} \alpha(y), \beta^{n} \alpha(x)\right\rangle_{\beta^{n}} \\
& =\beta^{n}\left(\beta^{2 n} \alpha^{2}(x) \cdot \beta^{2 n}(\langle y ; z, t\rangle)\right)-\beta^{n}\left(\beta^{2 n} \alpha^{2}(y) \cdot \beta^{2 n}(\langle x ; z, t\rangle)\right) \\
& +\beta^{2 n}\left(\left\langle\beta^{n}(y \cdot x) ; \beta^{n} \alpha(z), \beta^{n} \alpha(t)\right\rangle\right)+\beta^{n}\left(\beta^{2 n} \alpha^{2}(t) \cdot \beta^{2 n}(\langle z ; y, x\rangle)\right) \\
& -\beta^{n}\left(\beta^{2 n} \alpha^{2}(z) \cdot \beta^{2 n}(\langle t ; y, x\rangle)\right)+\beta^{2 n}\left(\left\langle\beta^{n}(z \cdot t) ; \beta^{n} \alpha(y), \beta^{n} \alpha(x)\right\rangle\right) \\
& =\beta^{3 n}\left(\alpha^{2}(x) \cdot\langle y ; z, t\rangle\right)-\beta^{3 n}\left(\alpha^{2}(y) \cdot\langle x ; z, t\rangle\right)+\beta^{3 n}(\langle y \cdot x ; \alpha(z), \alpha(t)\rangle) \\
& +\beta^{3 n}\left(\alpha^{2}(t) \cdot\langle z ; y, x\rangle\right)-\beta^{3 n}\left(\alpha^{2}(z) \cdot\langle t ; y, x\rangle\right)+\beta^{3 n}(\langle z \cdot t ; \alpha(y), \alpha(x)\rangle) \\
& =\beta^{3 n}\left(\alpha^{2}(x) \cdot\langle y ; z, t\rangle-\alpha^{2}(y) \cdot\langle x ; z, t\rangle+\langle y \cdot x ; \alpha(z), \alpha(t)\rangle\right. \\
& \left.+\alpha^{2}(t) \cdot\langle z ; y, x\rangle-\alpha^{2}(z) \cdot\langle t ; y, x\rangle+\langle z \cdot t ; \alpha(y), \alpha(x)\rangle\right) \\
& =0 \text { by }\left(\mathrm{HH}_{14}\right) \text { for } V_{\alpha} \text {; } \\
& \left(\beta^{n} \alpha\right)^{2}(y) *_{\beta^{n}}\langle x ; z, t\rangle_{\beta^{n}}-\left(\beta^{n} \alpha\right)^{2}(x) *_{\beta^{n}}\langle y ; z, t\rangle_{\beta^{n}} \\
& +\left(\beta^{n} \alpha\right)^{2}(z) *_{\beta^{n}}\langle t ; y, x\rangle_{\beta^{n}}-\left(\beta^{n} \alpha\right)^{2}(t) *_{\beta^{n}}\langle z ; y, x\rangle_{\beta^{n}} \\
& =\beta^{n}\left(\beta^{2 n} \alpha^{2}(y) * \beta^{2 n}(\langle x ; z, t\rangle)\right)-\beta^{n}\left(\beta^{2 n} \alpha^{2}(x) * \beta^{2 n}(\langle y ; z, t\rangle)\right) \\
& +\beta^{n}\left(\beta^{2 n} \alpha^{2}(z) * \beta^{2 n}(\langle t ; y, x\rangle)-\beta^{n}\left(\beta^{n} \alpha^{2 n}(t) * \beta^{2 n}(\langle z ; y, x\rangle)\right)\right. \\
& =\beta^{3 n}\left(\alpha^{2}(y) *\langle x ; z, t\rangle-\alpha^{2}(x) *\langle y ; z, t\rangle+\alpha^{2}(z) *\langle t ; y, x\rangle\right. \\
& \left.-\alpha^{2}(t) *\langle z ; y, x\rangle\right) \\
& =0 \text { by }\left(\mathrm{HH}_{15}\right) \text { for } V_{\alpha} \text {; }
\end{aligned}
$$




$$
\begin{aligned}
& \sum\left\{\left\langle\left(\left\langle z \cdot \beta^{n} t ; \beta^{n} \alpha(y), \beta^{n} \alpha(x)\right\rangle_{\beta^{n}}+\left(\beta^{n} \alpha\right)^{2}(t) \cdot \beta^{n}\langle z ; y, x\rangle_{\beta^{n}}\right.\right.\right. \\
& \left.\left.-\left(\beta^{n} \alpha\right)^{2}(z) \cdot \beta^{n}\langle t ; y, x\rangle_{\beta^{n}}\right) ;\left(\beta^{n} \alpha\right)^{3}(u),\left(\beta^{n} \alpha\right)^{3}(v)\right\rangle_{\beta^{n}} \\
& +\left\langle\left(\beta^{n} \alpha\right)^{2}(u) \cdot \beta^{n}\left(\beta^{n} \alpha\right)^{2}(v) ;\left\langle\beta^{n} \alpha(t) ; \beta^{n} \alpha(y), \beta^{n} \alpha(x)\right\rangle_{\beta^{n}},\left(\beta^{n} \alpha\right)^{3}(z)\right\rangle_{\beta^{n}} \\
& +\left(\beta^{n} \alpha\right)^{4}(v) \cdot \beta^{n}\left\langle\left(\beta^{n} \alpha\right)^{2}(u) ;\langle t ; y, x\rangle_{\beta^{n}},\left(\beta^{n} \alpha\right)^{2}(z)\right\rangle_{\beta^{n}} \\
& -\left(\beta^{n} \alpha\right)^{4}(u) \cdot \beta^{n}\left\langle\left(\beta^{n} \alpha\right)^{2}(v) ;\langle t ; y, x\rangle_{\beta^{n}},\left(\beta^{n} \alpha\right)^{2}(z)\right\rangle_{\beta^{n}} \\
& -\left(\left\langle\left(\beta^{n} \alpha\right)^{2}(u) \cdot \beta_{\beta^{n}}\left(\beta^{n} \alpha\right)^{2}(v) ;\left\langle\beta^{n} \alpha(z) ; \beta^{n} \alpha(y), \beta^{n} \alpha(x)\right\rangle_{\beta^{n}},\left(\beta^{n} \alpha\right)^{3}(t)\right\rangle_{\beta^{n}}\right. \\
& +\left(\beta^{n} \alpha\right)^{4}(v) \cdot \beta^{n}\left\langle\left(\beta^{n} \alpha\right)^{2}(u) ;\langle z ; y, x\rangle_{\beta^{n}},\left(\beta^{n} \alpha\right)^{2}(t)\right\rangle_{\beta^{n}} \\
& \left.\left.-\left(\beta^{n} \alpha\right)^{4}(u) \cdot \beta^{n}\left\langle\left(\beta^{n} \alpha\right)^{2}(v) ;\langle z ; y, x\rangle_{\beta^{n}},\left(\beta^{n} \alpha\right)^{2}(t)\right\rangle_{\beta^{n}}\right)\right\} \\
& =\sum\left\{\beta ^ { 2 n } \left(\left\langle\left(\beta^{2 n}\left(\left\langle\beta^{n}(z \cdot t) ; \beta^{n} \alpha(y), \beta^{n} \alpha(x)\right\rangle\right)+\beta^{n}\left(\beta^{2 n} \alpha^{2}(t) \cdot \beta^{2 n}(\langle z ; y, x\rangle)\right)\right.\right.\right.\right. \\
& \left.\left.\left.-\beta^{n}\left(\beta^{2 n} \alpha^{2}(z) \cdot \beta^{2 n}(\langle t ; y, x\rangle)\right)\right) ; \beta^{3 n} \alpha^{3}(u), \beta^{3 n} \alpha^{3}(v)\right\rangle\right) \\
& +\beta^{2 n}\left(\left\langle\beta^{n}\left(\beta^{2 n} \alpha^{2}(u) \cdot \beta^{2 n} \alpha^{2}(v)\right) ; \beta^{2 n}\left(\left\langle\beta^{n} \alpha(t) ; \beta^{n} \alpha(y),\right.\right.\right.\right. \\
& \left.\left.\left.\left.\beta^{n} \alpha(x)\right\rangle\right), \beta^{3 n} \alpha^{3}(z)\right\rangle\right) \\
& +\beta^{n}\left(\beta^{4 n} \alpha^{4}(v) \cdot \beta^{2 n}\left(\left\langle\beta^{2 n} \alpha^{2}(u) ; \beta^{2 n}(\langle t ; y, x\rangle), \beta^{2 n} \alpha^{2}(z)\right\rangle\right)\right) \\
& -\beta^{n}\left(\beta^{4 n} \alpha^{4}(u) \cdot \beta^{2 n}\left(\left\langle\beta^{2 n} \alpha^{2}(v) ; \beta^{2 n}(\langle t ; y, x\rangle), \beta^{2 n} \alpha^{2}(z)\right\rangle\right)\right) \\
& -\left(\beta ^ { 2 n } \left(\left\langle\beta^{n}\left(\beta^{2 n} \alpha^{2}(u) \cdot \beta^{2 n} \alpha^{2}(v)\right) ; \beta^{2 n}\left(\left\langle\beta^{n} \alpha(z) ; \beta^{n} \alpha(y),\right.\right.\right.\right.\right. \\
& \left.\left.\left.\beta^{n} \alpha(x)\right\rangle\right),\left(\beta^{3 n} \alpha^{3}(t)\right\rangle\right) \\
& +\beta^{n}\left(\beta^{4 n} \alpha^{4}(v) \cdot \beta^{2 n}\left(\left\langle\beta^{2 n} \alpha^{2}(u) ; \beta^{2 n}(\langle z ; y, x\rangle), \beta^{2 n} \alpha^{2}(t)\right\rangle\right)\right) \\
& \left.\left.-\beta^{n}\left(\beta^{4 n} \alpha^{4}(u) \cdot \beta^{2 n}\left(\left\langle\beta^{2 n} \alpha^{2}(v) ; \beta^{2 n}(\langle z ; y, x\rangle), \beta^{2 n} \alpha^{2}(t)\right\rangle\right)\right)\right)\right\}
\end{aligned}
$$




$$
\begin{aligned}
&=\sum\left\{\beta ^ { 2 n } \left(\left\langle\beta^{3 n}(\langle z \cdot t ; \alpha(y), \alpha(x)\rangle)+\beta^{3 n}\left(\alpha^{2}(t) \cdot\langle z ; y, x\rangle\right)\right.\right.\right. \\
&\left.\left.\left.-\beta^{3 n}\left(\alpha^{2}(z) \cdot\langle t ; y, x\rangle\right)\right) ; \beta^{3 n} \alpha^{3}(u), \beta^{3 n} \alpha^{3}(v)\right\rangle\right) \\
&+\beta^{2 n}\left(\left\langle\beta^{3 n}\left(\alpha^{2}(u) \cdot \alpha^{2}(v)\right) ; \beta^{3 n}(\langle\alpha(t) ; \alpha(y), \alpha(x)\rangle), \beta^{3 n} \alpha^{3}(z)\right\rangle\right) \\
&+\beta^{n}\left(\beta^{4 n} \alpha^{4}(v) \cdot \beta^{4 n}\left(\left\langle\alpha^{2}(u) ;\langle t ; y, x\rangle, \alpha^{2}(z)\right\rangle\right)\right) \\
&-\beta^{n}\left(\left\langle\beta^{4 n} \alpha^{4}(u) \cdot \beta^{4 n}\left(\left\langle\alpha^{2}(v) ;\langle t ; y, x\rangle, \alpha^{2}(z)\right\rangle\right)\right)\right. \\
&-\left(\beta^{2 n}\left(\left\langle\beta^{3 n}\left(\alpha^{2}(u) \cdot \alpha^{2}(v)\right) ; \beta^{3 n}(\langle\alpha(z) ; \alpha(y), \alpha(x)\rangle), \beta^{3 n} \alpha^{3}(t)\right\rangle\right)\right. \\
&+\beta^{n}\left(\beta^{4 n} \alpha^{4}(v) \cdot \beta^{4 n}\left(\left\langle\alpha^{2}(u) ;\langle z ; y, x\rangle, \alpha^{2}(t)\right\rangle\right)\right) \\
&\left.\left.-\beta^{n}\left(\beta^{4 n} \alpha^{4}(u) \cdot \beta^{4 n}\left(\left\langle\alpha^{2}(v) ;\langle z ; y, x\rangle, \alpha^{2}(t)\right\rangle\right)\right)\right)\right\} \\
&=\beta^{5 n}\left(\sum\left\{\left\langle\left(\langle z \cdot t ; \alpha(y), \alpha(x)\rangle+\alpha^{2}(t) \cdot\langle z ; y, x\rangle-\alpha^{2}(z) \cdot\langle t ; y, x\rangle\right) ; \alpha^{3}(u), \alpha^{3}(v)\right\rangle\right)\right. \\
&+\left\langle\alpha^{2}(u) \cdot \alpha^{2}(v) ;\langle\alpha(t) ; \alpha(y), \alpha(x)\rangle, \alpha^{3}(z)\right\rangle \\
&+\alpha^{4}(v) \cdot\left\langle\alpha^{2}(u) ;\langle t ; y, x\rangle, \alpha^{2}(z)\right\rangle \\
&\left.\left.\left.\left.+\alpha^{4}(u) \cdot\left\langle\alpha^{2}(v) ;\langle z ; y, x\rangle, \alpha^{2}(t)\right\rangle\right)\right)\right\}\right) \\
&+\alpha^{4}(u) \cdot\left\langle\alpha^{2}(v) ;\langle t ; y, x\rangle, \alpha^{2}(z)\right\rangle \\
&-\left(\left\langle\alpha^{2}(u) \cdot \alpha^{2}(v) ;\langle\alpha(z) ; \alpha(y), \alpha(x)\rangle, \alpha^{3}(t)\right\rangle\right. \\
& \alpha^{4}(v) \cdot\left\langle\left(\alpha^{2}(u) ;\langle z ; y, x\rangle, \alpha^{2}(t)\right\rangle\right. \\
& \text { for } V_{\alpha} ; \\
&
\end{aligned}
$$




$$
\begin{aligned}
& \sum\left\{\left(\left\langle\left(\beta^{n} \alpha\right)^{2}(v) ;\langle t ; y, x\rangle_{\beta^{n}},\left(\beta^{n} \alpha\right)^{2}(z)\right\rangle_{\beta^{n}}\right.\right. \\
& \left.-\left\langle\left(\beta^{n} \alpha\right)^{2}(v) ;\langle z ; y, x\rangle_{\beta^{n}},\left(\beta^{n} \alpha\right)^{2}(t)\right\rangle_{\beta^{n}}\right) *_{\beta^{n}}\left(\beta^{n} \alpha\right)^{4}(u) \\
& +\left(\left\langle\left(\beta^{n} \alpha\right)^{2}(u) ;\langle z ; y, x\rangle_{\beta^{n}},\left(\beta^{n} \alpha\right)^{2}(t)\right\rangle_{\beta^{n}}\right. \\
& \left.\left.-\left\langle\left(\beta^{n} \alpha\right)^{2}(u) ;\langle t ; y, x\rangle_{\beta^{n}},\left(\beta^{n} \alpha\right)^{2}(z)\right\rangle_{\beta^{n}}\right) *_{\beta^{n}}\left(\beta^{n} \alpha\right)^{4}(v)\right\} \\
& =\sum\left\{\beta ^ { n } \left(\left(\beta^{2 n}\left(\left\langle\beta^{2 n} \alpha^{2}(v) ; \beta^{2 n}(\langle t ; y, x\rangle), \beta^{2 n} \alpha^{2}(z)\right\rangle\right)\right.\right.\right. \\
& \left.\left.-\beta^{2 n}\left(\left\langle\beta^{2 n} \alpha^{2}(v) ; \beta^{2 n}(\langle z ; y, x\rangle), \beta^{2 n} \alpha^{2}(t)\right\rangle\right)\right) * \beta^{4 n} \alpha^{4}(u)\right) \\
& +\beta^{n}\left(\left(\beta^{2 n}\left(\left\langle\beta^{2 n} \alpha^{2}(u) ; \beta^{2 n}(\langle z ; y, x\rangle), \beta^{2 n} \alpha^{2}(t)\right\rangle\right)\right.\right. \\
& \left.\left.\left.\left.-\beta^{2 n}\left(\left\langle\beta^{2 n} \alpha^{2}(u) ; \beta^{2 n}(\langle t ; y, x\rangle), \beta^{2 n} \alpha\right)^{2}(z)\right\rangle\right)\right) * \beta^{4 n} \alpha^{4}(v)\right)\right\} \\
& =\sum\left\{\beta ^ { n } \left(\left(\beta^{4 n}\left(\left\langle\alpha^{2}(v) ;\langle t ; y, x\rangle, \alpha^{2}(z)\right\rangle\right)\right.\right.\right. \\
& \left.\left.-\beta^{4 n}\left(\left\langle\alpha^{2}(v) ;\langle z ; y, x\rangle, \alpha^{2}(t)\right\rangle\right)\right) * \beta^{4 n} \alpha^{4}(u)\right) \\
& +\beta^{n}\left(\left(\beta^{4 n}\left(\left\langle\alpha^{2}(u) ;\langle z ; y, x\rangle, \alpha^{2}(t)\right\rangle\right)\right.\right. \\
& \left.\left.\left.\left.-\beta^{4 n}\left(\left\langle\alpha^{2}(u) ;\langle t ; y, x\rangle, \alpha\right)^{2}(z)\right\rangle\right)\right) * \beta^{4 n} \alpha^{4}(v)\right)\right\} \\
& =\beta^{5 n}\left(\sum \left\{\left(\left\langle\alpha^{2}(v) ;\langle t ; y, x\rangle, \alpha^{2}(z)\right\rangle-\left\langle\alpha^{2}(v) ;\langle z ; y, x\rangle, \alpha^{2}(t)\right\rangle\right) * \alpha^{4}(u)\right.\right. \\
& \left.\left.+\left(\left\langle\alpha^{2}(u) ;\langle z ; y, x\rangle, \alpha^{2}(t)\right\rangle-\left\langle\alpha^{2}(u) ;\langle t ; y, x\rangle, \alpha^{2}(z)\right\rangle\right) * \alpha^{4}(v)\right\}\right) \\
& =0 \text { by }\left(\mathrm{HH}_{17}\right) \text { for } V_{\alpha} \text {; }
\end{aligned}
$$




$$
\begin{aligned}
& \sum\left\{\left\langle\left(\beta^{n} \alpha\right)^{4}(w) ;\left(\left\langle\left(\beta^{n} \alpha\right)^{2}(v) ;\langle t ; y, x\rangle_{\beta^{n}},\left(\beta^{n} \alpha\right)^{2}(z)\right\rangle_{\beta^{n}}\right.\right.\right. \\
& \left.\left.-\left(\left\langle\beta^{n} \alpha\right)^{2}(v) ;\langle z ; y, x\rangle_{\beta^{n}},\left(\beta^{n} \alpha\right)^{2}(t)\right\rangle_{\beta^{n}}\right),\left(\beta^{n} \alpha\right)^{4}(u)\right\rangle_{\beta^{n}} \\
& +\left(\left\langle\beta^{n} \alpha\right)^{4}(w) ;\left(\left\langle\left(\beta^{n} \alpha\right)^{2}(u) ;\langle z ; y, x\rangle_{\beta^{n}},\left(\beta^{n} \alpha\right)^{2}(t)\right\rangle_{\beta^{n}}\right.\right. \\
& \left.\left.\left.-\left\langle\left(\beta^{n} \alpha\right)^{2}(u) ;\langle t ; y, x\rangle_{\beta^{n}},\left(\beta^{n} \alpha\right)^{2}(z)\right\rangle_{\beta^{n}}\right),\left(\beta^{n} \alpha\right)^{4}(v)\right\rangle_{\beta^{n}}\right\} \\
& =\sum\left\{\beta ^ { 2 n } \left(\left\langle\beta^{4 n} \alpha^{4}(w) ;\left(\beta^{2 n}\left(\left\langle\beta^{2 n} \alpha^{2}(v) ; \beta^{2 n}(\langle t ; y, x\rangle), \beta^{2 n} \alpha^{2}(z)\right\rangle\right)\right.\right.\right.\right. \\
& \left.\left.\left.-\beta^{2 n}\left(\left\langle\beta^{2 n} \alpha^{2}(v) ; \beta^{2 n}(\langle z ; y, x\rangle), \beta^{2 n} \alpha^{2}(t)\right\rangle\right)\right), \beta^{4 n} \alpha^{4}(u)\right\rangle\right) \\
& +\beta^{2 n}\left(\left\langle\beta^{4 n} \alpha\right)^{4}(w) ;\left(\beta^{2 n}\left(\left\langle\beta^{2 n} \alpha^{2}(u) ; \beta^{2 n}(\langle z ; y, x\rangle), \beta^{2 n} \alpha^{2}(t)\right\rangle\right)\right.\right. \\
& \left.\left.\left.\left.-\beta^{2 n}\left(\left\langle\beta^{2 n} \alpha^{2}(u) ; \beta^{2 n}(\langle t ; y, x\rangle), \beta^{2 n} \alpha^{2}(z)\right\rangle\right)\right), \beta^{4 n} \alpha^{4}(v)\right\rangle\right)\right\} \\
& =\sum\left\{\beta ^ { 2 n } \left(\left\langle\beta^{4 n} \alpha^{4}(w) ;\left(\beta^{4 n}\left(\left\langle\alpha^{2}(v) ;\langle t ; y, x\rangle, \alpha^{2}(z)\right\rangle\right)\right.\right.\right.\right. \\
& \left.\left.\left.-\beta^{4 n}\left(\left\langle\alpha^{2}(v) ;\langle z ; y, x\rangle, \alpha^{2}(t)\right\rangle\right)\right), \beta^{4 n} \alpha^{4}(u)\right\rangle\right) \\
& +\beta^{2 n}\left(\left\langle\beta^{4 n} \alpha\right)^{4}(w) ;\left(\beta^{4 n}\left(\left\langle\alpha^{2}(u) ;\langle z ; y, x\rangle, \alpha^{2}(t)\right\rangle\right)\right.\right. \\
& \left.\left.\left.\left.-\beta^{4 n}\left(\left\langle\alpha^{2}(u) ;\langle t ; y, x\rangle, \alpha^{2}(z)\right\rangle\right)\right), \beta^{4 n} \alpha^{4}(v)\right\rangle\right)\right\} \\
& =\beta^{6 n}\left(\sum \left\{\left\langle\alpha^{4}(w) ;\left(\left\langle\alpha^{2}(v) ;\langle t ; y, x\rangle, \alpha^{2}(z)\right\rangle\right.\right.\right.\right. \\
& \left.\left.-\left\langle\alpha^{2}(v) ;\langle z ; y, x\rangle, \alpha^{2}(t)\right\rangle\right), \alpha^{4}(u)\right\rangle \\
& +\langle\alpha)^{4}(w) ;\left(\left\langle\alpha^{2}(u) ;\langle z ; y, x\rangle, \alpha^{2}(t)\right\rangle\right. \\
& \left.\left.\left.\left.-\left\langle\alpha^{2}(u) ;\langle t ; y, x\rangle, \alpha^{2}(z)\right\rangle\right), \alpha^{4}(v)\right\rangle\right\}\right) \\
& =0 \text { by }\left(\mathrm{HH}_{18}\right) \text { for } V_{\alpha} \text {. }
\end{aligned}
$$

Therefore, we prove the validity for $V_{\beta^{n}}$ of the set of identities of type $\left(\mathrm{HH}_{1}\right)-\left(\mathrm{HH}_{18}\right)$ and so we get that $V_{\beta^{n}}$ is a Hom-hyporeductive triple algebra. This finishes the proof. 
From the Theorem 3.3, we get the following:

Corollary 3.4. Let $(V, \star, *,\langle;\rangle,, \beta)$ be a Hom-hyporeductive triple algebra. Then for each $n \geq 0$, the Hom-algebra $V_{(n)}=\left(V, \star{ }_{(n)}=\beta^{n} \circ \star\right.$, $\left.*_{(n)}=\beta^{n} \circ *,\langle;,\rangle_{(n)}=\beta^{2 n} \circ\langle;\rangle,, \beta^{n+1}\right)$ is a Hom-hyporeductive triple algebra.

Proof. We get the proof from the one of Theorem 3.3 if set $\alpha=\beta$.

Corollary 3.5. Let $(V, \cdot, *,\langle;\rangle$,$) be a hyporeductive triple algebra and$ $\beta$ a morphism of $(V, \cdot, *,\langle;\rangle$,$) . If define on V$ the operations: $x \cdot \beta,=$ $(x \cdot y), x *_{\beta} y:=\beta(x * y)$ and $\langle x ; y, z\rangle_{\beta}:=\beta^{2}(\langle x ; y, z\rangle)$, for all $x, y, z$ in $V$. Then $V_{\beta}=\left(V, \cdot_{\beta}, *_{\beta},\langle;,\rangle_{\beta}, \beta\right)$ is a Hom-hyporeductive triple algebra.

Proof. The proof follows from the one of Theorem 3.3 for $\alpha=I d$ and $n=1$.

Observe that Corollary 3.5 gives a method for constructing a Homhyporeductive triple algebra from a hyporeductive triple algebra. This is an extension of a result due to Yau [28] giving a general construction method of Hom-algebras from their corresponding untwisted version (see $[3,4,5,10,30]$ for a use for such an extension).

We have also the following Theorem which shows that Homhyporeductive triple algebras are closed under taking derived binaryternary Hom-algebras.

Theorem 3.6. Let $(V, \star, *,\langle;\rangle,, \beta)$ be a Hom-hyporeductive triple algebra. Then for each $n \geq 0$, the $n$-th derived binary ternary Hom-algebra $V^{(n)}=\left(V, \star^{(n)}=\beta^{2^{n}-1} \circ, \star, *{ }^{(n)}=\beta^{2^{n}-1} \circ *,\langle;,\rangle^{(n)}=\beta^{2^{n}-1} \circ\langle;\rangle,, \beta^{2^{n}}\right)$ is also a Hom-hyporeductive triple algebra. 
Proof. Since $V^{(0)}=V, V^{(1)}=\left(V, \star^{(1)}=\beta \circ \star, *^{(1)}=\beta \circ *,\langle;,\rangle^{(1)}=\right.$ $\left.\beta^{2} \circ\langle;\rangle,, \beta\right)=V_{(1)} \quad\left(\right.$ see Corollary 3.4) and $V^{(n+1)}=\left(V^{(n)}\right)^{(1)}$, by an induction argument, it suffices to prove the case $n=1$ which follows from the proof of Corollary 3.4.

\section{Examples}

In this section, we give some examples of Hom-hyporeductive triple algebras. We do not classify all algebra morphisms of all the real 2-dimensional hyporeductive triple algebras considered and subsequently real 2-dimensional Hom-hyporeductive triple algebras are not classified. Such classification remains then an open problem. We consider only non trivial cases, i.e., we omit mentioning Hom-Lie triple algebras (i.e., HomLie-Yamaguti algebras) and Hom-Bol algebras and the o-map as twisting map since it is always an algebra morphism and it gives rise to the zero Hom-algebra. If $(V, \cdot, *,\langle;\rangle$,$) is a 2$-dimensional hyporeductive algebra with basis $(u, v)$, then a linear map $\alpha: V \rightarrow V$ defined by $\alpha(u)=x u+$ $y v, \alpha(v)=z u+t v$ is a self-morphism of $(V, \cdot, *,\langle;\rangle$,$) if and only if$

$$
\begin{aligned}
\alpha(u \cdot v)=\alpha(u) \cdot \alpha(v), \alpha(u * v) & =\alpha(u) * \alpha(v), \alpha(\langle w ; u, v\rangle) \\
& =\langle\alpha(w) ; \alpha(u), \alpha(v)\rangle .
\end{aligned}
$$

It is proved ([11], Theorem 2.4 and Corollary 2.6) that any non trivial 2 -dimensional hyporeductive triple algebra over the field of real numbers is isomorphic to only one of the algebras described below:

$$
\begin{aligned}
&\left(\mathrm{V}_{1}\right) u \cdot v=a u, u * v=c u+d v,\langle u ; u, v\rangle=0,\langle v ; u, v\rangle \\
&=k u,(c, d) \neq(0,0), \\
&\left(\mathrm{V}_{2}\right) u \cdot v=a u+b v, u * v=c u+d v,\langle u ; u, v\rangle=0,\langle v ; u, v\rangle=0, \\
&(a \neq 0, b \neq 0,(c, d) \neq(0,0)),
\end{aligned}
$$




$$
\begin{array}{r}
\left(\mathrm{V}_{3}\right) u \cdot v=a u+b v, u * v=c u+d v,\langle u ; u, v\rangle=e u+f v \\
\langle v ; u, v\rangle=k u-e v,(a \neq 0, b \neq 0, e \neq 0, f \neq 0, k \neq 0, \\
(c, d) \neq(0,0) \text { af }-b e=0=b k+a e) .
\end{array}
$$

Now, with the linear map $\alpha$ given as above, we shall discuss the condition (7) for each of the types $\left(\mathrm{V}_{1}\right),\left(\mathrm{V}_{2}\right)$, and $\left(\mathrm{V}_{3}\right)$.

- Case of the type $\left(\mathrm{V}_{1}\right)$ :

For this type, conditions (7) lead to the following simultaneous constraints on the coefficients $x, y, z, t$ of $\alpha: x(1-t)=x c(1-t)+d z=d t$ $(1-x)=k x\left(1-t^{2}\right)=y=0$. If $x=0$, from the equations above, we see that non-zero morphism $\alpha$ is defined as

$$
\alpha(u)=0, \alpha(v)=z u+t v,(\text { with }(z, t) \neq(0,0)),
$$

and, applying Corollary 3.5, we get that $\left(V_{1}\right)$ is twisted into the Homhyporeductive triple algebra $\left(V,{ }_{\alpha}, *_{\alpha},\langle;,\rangle_{\alpha}, \alpha\right)$ :

$$
\begin{gathered}
u \cdot \alpha_{\alpha} v=0, u *_{\alpha} v=(d z) u+(d t) v,\langle u ; u, v\rangle_{\alpha}=0,\langle v ; u, v\rangle_{\alpha}=0, \\
((z, t) \neq(0,0)),
\end{gathered}
$$

if $x \neq 0$, then the equation $x(1-t)=0$ (see above) implies $t=1$ and then the morphism $\alpha$ is defined as

$$
\alpha(u)=x u, \alpha(v)=z u+v .
$$

Notice that this morphism is not the identity map if and only $(x, z) \neq(1,0)$. Then the application of Corollary 3.5 gives rise to the Hom-hyporeductive triple algebra $\left(V, \cdot \alpha, *_{\alpha},\langle;,\rangle_{\alpha}, \alpha\right)$ :

$$
\begin{array}{r}
u \cdot{ }_{\alpha} v=(x a) u, u *_{\alpha} v=(c x+d z) u+d v,\langle u ; u, v\rangle_{\alpha}=0, \\
\langle v ; u, v\rangle_{\alpha}=\left(k x^{2}\right) u .
\end{array}
$$


- Case of the type $\left(\mathrm{V}_{2}\right)$ :

For this type, conditions (7) lead to the following simultaneous constraints on the coefficients $x, y, z, t$ for $\alpha: a(x-x t+y z)+b z=c$ $(x-x t+y z)+d z=a y+b(t-x t+y z)=c y+d(t-x t+y z)=0$.

We see that These constraints are very difficult to be solved. Two particular non-zero morphisms are given by

$$
\begin{aligned}
& \alpha(u)=x u, \alpha(v)=0, x \neq 0, \\
& \alpha(u)=u, \alpha(v)=y v, y \in \mathbb{R} .
\end{aligned}
$$

Notice that the morphism defined in (10) is not the identity map and the one defined in (11) is not the identity map if and only if $y \neq 1$.

For the morphism defined in (10), the application of Corollary 3.5 gives rise to the Hom-hyporeductive triple algebra $\left(V,{ }_{\alpha}, *_{\alpha},\langle;,\rangle_{\alpha}, \alpha\right)$ :

$$
u \cdot{ }_{\alpha} v=(x a) u, u *_{\alpha} v=(c x) u,\langle u ; u, v\rangle_{\alpha}=\langle u ; u, v\rangle_{\alpha}=0 .
$$

For the morphism defined in (11), the application of Corollary 3.5 gives rise to the Hom-hyporeductive triple algebra $\left(V,{ }_{\alpha},{ }^{*} \alpha,\langle;,\rangle_{\alpha}, \alpha\right)$ :

$$
u \cdot{ }_{\alpha} v=a u+(b y) v, u *_{\alpha} v=c u+(d y) v,\langle u ; u, v\rangle_{\alpha}=\langle v ; u, v\rangle_{\alpha}=0 .
$$


- Case of the type $\left(\mathrm{V}_{3}\right)$;

For this type, conditions (7) lead to the following simultaneous constraints on the coefficients

$$
\left\{\begin{aligned}
a(x-x t+y z)+b z=c(x-x t+y z)+ & d z=a y+b(t-x t+y z) \\
= & c y+d(t-x t+y z)=0 \\
e\left(x-x^{2} t+x y z\right)+f z+k\left(z y^{2}-x y t\right)= & e\left(y+x y t-z y^{2}\right) \\
& +f\left(t-t x^{2}+x y z\right)=0 \\
e\left(-t+x t^{2}-y z t\right)+f\left(-x y t+y z^{2}\right)= & e\left(-z-x z t+y z^{2}\right) \\
& +k\left(x-x t^{2}+y z t\right)=0
\end{aligned}\right.
$$

where the unknows are $x, y, z, t$ and $a, b, c, d, e, f, k$ are parameters and satisfy $(a \neq 0, b \neq 0, e \neq 0, f \neq 0, k \neq 0,(c, d) \neq(0,0), a f-b e=0$ $=b k+a e)$.

Therefore linear maps of $\left(\mathrm{V}_{3}\right)$ satisfying the above system are morphisms of $\left(\mathrm{V}_{3}\right)$. Thus with these morphisms, the application of Corollary 3.5 allows to twist $\left(\mathrm{V}_{3}\right)$ into Hom-hyporeductive triple algebras.

\section{References}

[1] M. A. Akivis, Local differential quasigroups and 3-webs of multidimensional surfaces, In: Studies in the Theory of Quasigroups and Loops, Stiinta, Kishinev, Russian, 1973, 3-12.

[2] M. A. Akivis, Local algebras of a multidimensional 3-web, Siberian Mathematical Journal 17(7) (1976), 3-8.

DOI: https://doi.org/10.1007/BF00969285

[3] H. Ataguema, A. Makhlouf and S. D. Silvestrov, Generalization of $n$-ary Nambu algebras and beyond, Journal of Mathematical Physics 50(8) (2009); Article 083501.

DOI: https://doi.org/10.1063/1.3167801 
[4] S. Attan and A. N. Issa, Hom-Bol algebras, Quasigroups and Related Systems 21(2) (2013), 131-146.

[5] D. Gaparayi and A. N. Issa, A twisted generalization of Lie-Yamaguti algebras, International Journal of Algebra 6(7) (2012), 339-352.

[6] E. G. Goodaire, Alternative rings of small order and the hunt for Moufang circle loops in Nonassociative Algebra and its Applications (São Paulo, 1998), vol. 211 of Lecture Notes in Pure and Appl. Math., Marcel Dekker, New York, 2000, 137-146.

[7] J. T. Hartwig, D. Larsson and S. D. Silvestrov, Deformations of Lie algebras using $\sigma$-derivations, Journal of Algebra 295(2) (2006), 314-361.

DOI: https://doi.org/10.1016/j.jalgebra.2005.07.036

[8] I. R. Hentzel and L. A. Peresi, Special identities for Bol algebras, Linear Algebra and its Applications 436(7) (2012), 2315-2330.

DOI: https://doi.org/10.1016/j.laa.2011.09.021

[9] K. H. Hofmann and K. Strambach, Lie's fundamental theorems for local analytical loops, Pacific Journal of Mathematics 123(2) (1986), 301-327.

[10] A. N. Issa, Hom-Akivis algebras, Commentationes Mathematicae Universitatis Carolinae 52(4) (2011), 485-500.

[11] A. N. Issa, Classifying two-dimensional hyporeductive triple algebras, International Journal of Mathematics and Mathematical Sciences, 2006, 1-10; Article ID 013527.

DOI: https://doi.org/10.1155/IJMMS/2006/13527

[12] A. N. Issa, On the theory of hyporeductive algebras, Algebraic Methods in Geometry (V. V. Ryzhkov, et al., Editors) Collect. Sci. Woorks, Izd. Ross. Univ. druzhby Narodov, Moskva, 1992, pp. 20-25.

[13] A. N. Issa, Notes on the geometry of smooth hyporeductive loops, Algebras, Groups and Geometries 12(3) (1995), 223-246.

[14] A. N. Issa, Remarks on the construction of Lie-Yamaguti algebras, International Journal of Algebra 5(14) (2011), 667-677.

[15] D. Larsson and S. D. Silvestrov, Quasi-Hom-Lie algebras, central extensions and 2-cycle-like identifies, Journal of Algebra 288(2) (2005), 321-344.

DOI: https://doi.org/10.1016/j.jalgebra.2005.02.032

[16] D. Larsson and S. D. Silvestrov, Quasi-Lie algebras, Contemporary Mathematics 391 (2005).

[17] W. G. Lister, A structure theory of Lie triple system, Transactions of the American Mathematical Society 72(2) (1952), 217-242.

DOI: https://doi.org/10.2307/1990753

[18] A. Makhlouf, Hom-alternative algebras and Hom-Jordan algebras, International Electronic Journal of Algebra 8 (2010), 177-190. 
[19] A. Makhlouf and S. D. Silvestrov, Hom-algebra structures, Journal of Generalized Lie Theory and Applications 2(2) (2008), 51-64.

[20] P. O. Mikheev, Commutator algebras of right alternative algebras (Russian), Mat. Issled. 113 (1990), 62-65.

[21] P. O. Mikheev and L. V. Sabinin, Quasigroups and differential geometry, Quasigroups and Loops: Theory and Applications (O. Chein, H. O. Pflugfelder and J. D. H. Smith, Editors), Sigma Series in Pure Mathematics, Vol. 8, Heldermann, Berlin (1990), pp. 357-430.

[22] L. V. Sabini, On smooth hyporeductive loops, Soviet Mathematics, Doklady 42(2) (1991), 524-526.

[23] L. V. Sabini, Smooth Hyporeductive Loops, Webs and Quasigroups (Moscow, 1989), Tver. Gos. Univ., Tver, 1991, pp. 129-137.

[24] L. V. Sabini, The theory of smooth hyporeductive and pseudoreductive loops, Algebras, Groups and Geometries 13(1) (1996), 1-24.

[25] A. A. Sagle, Malcev algebras, Transactions of the American Mathematical Society 101(3) (1961), 426-458.

DOI: https://doi.org/10.1090/S0002-9947-1961-0143791-X

[26] K. Yamaguti, On the Lie triple system and its generalisation, Journal of Science of the Hiroshima University, Series A 21(3) (1958), 155-160.

DOI: https://doi.org/10.32917/hmj/1555639527

[27] D. Yau, Hom-Maltsev, Hom-alternative, and Hom-Jordan algebras, International Electronic Journal of Algebras 11 (2012), 177-217.

[28] D. Yau, Hom-algebras and Homology, Journal of Lie Theory 19(2) (2009), 409-421.

[29] D. Yau, Hom-Novikov algebras, Journal of Physics A: Mathematical and Theoretical 44(8) (2011); Article 085202.

DOI: https://doi.org/10.1088/1751-8113/44/8/085202

[30] D. Yau, On $n$-ary Hom-Nambu and Hom-Nambu-Lie algebras, Journal of Geometry and Physics 62(2) (2012), 506-522.

DOI: https://doi.org/10.1016/j.geomphys.2011.11.006 